\title{
Mothering From the Inside Out: Results of a second randomized clinical trial testing a mentalization-based intervention for mothers in addiction treatment
}

\author{
NANCY E. SUCHMAN, ${ }^{a, b}$ CINDY L. DeCOSTE, ${ }^{a}$ THOMAS J. MCMAHON,${ }^{a, b}$ RACHEL DALTON, ${ }^{c}$

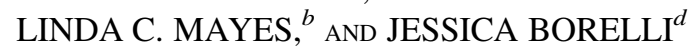 \\ ${ }^{a}$ Yale University School of Medicine; ${ }^{b}$ Yale Child Study Center; ${ }^{c}$ APT Foundation; and ${ }^{d}$ Pomona College
}

\begin{abstract}
Mothers with histories of alcohol and drug addiction have shown greater difficulty parenting young children than mothers with no history of substance misuse. This study was the second randomized clinical trial testing the efficacy of Mothering From the Inside Out (MIO), a 12-week mentalization-based individual therapy designed to address psychological deficits commonly associated with chronic substance use that also interfere with the capacity to parent young children. Eighty-seven mothers caring for a child between 11 and 60 months of age were randomly assigned to receive 12 sessions of MIO versus 12 sessions of parent education (PE), a psychoeducation active control comparison. Maternal reflective functioning, representations of caregiving, mother-child interaction quality, and child attachment were evaluated at baseline and posttreatment and 3-month follow-up. Mother-child interaction quality was assessed again at 12-month follow-up. In comparison with PE mothers, MIO mothers demonstrated a higher capacity for reflective functioning and representational coherence at posttreatment and 3-month follow-up. At 12-month follow-up, compared to PE cohorts, MIO mothers demonstrated greater sensitivity, their children showed greater involvement, and MIO dyads showed greater reciprocity. As addiction severity increased, MIO also appeared to serve as a protective factor for maternal reflective functioning, quality of mother-child interactions, and child attachment status. Results demonstrate the promise of mentalization-based interventions provided concomitant with addiction treatment for mothers and their young children.
\end{abstract}

Although quality of caregiving varies widely, as a group, mothers who have histories of chronic substance use are at greater risk than mothers with no substance use history for losing custody of their young children (Choi \& Ryan, 2007; Department of Health and Human Services, 1999; Grant et al., 2011). In developmental studies (e.g., Burns, Chetik, Burns, \& Clark, 1997; Hans, Bernstein, \& Henson, 1999), mothers with substance use disorders have also shown lower levels of sensitivity and responsiveness to their infant's cues and marked oscillation between intrusive, overcontrolling behaviors and passive withdrawal.

Although addiction severity among child-rearing women seeking treatment for drug addiction has increased signifi-

We acknowledge the National Institute on Drug Abuse for its ongoing funding for this research including R01 DA17294 (N.E.S., Principal Investigator) and K02 DA023504 (N.E.S., Principal Investigator). We thank our Research Assistants, Laura Ciarleglio, Laura Donald, Lourdes de las Heras Kuhn, Emily Pariseau, Hannah Rasmussen, Lauren Vega, and Mayra Vega, for their many important contributions to this work. We are grateful for the input we received from clinical consultants Susan Bers and Lauren Dennehy and guidance from the late Bruce Rounsaville and Patty Rosenberger. Finally, we thank the patients and clinicians at the APT Foundation without whose participation and support this work would not have been possible.

Address correspondence and reprint requests to: Nancy E. Suchman, Moms 'n' Kids Program, Yale Child Study Center, One Long Wharf Drive, Suite 310, New Haven, CT 06511; E-mail: nancy.suchman@yale.edu. cantly in recent years (Greenfield, Back, Lawson, \& Brady, 2010), addiction treatment programs rarely address parenting deficits. Moreover, parenting interventions designed for the general population (e.g., Triple P, Sanders, 1999; The Incredible Years, Webster-Stratton \& Reid, 2010) fail to address the parenting problems of parents with addictive disorders, who often drop out prematurely or require more intensive and extensive clinical efforts. Reviews of clinical trials testing psychoeducational parenting interventions with this vulnerable population have generally shown little to no efficacy for improving parenting behavior, parent-child relationships, or children's well-being (for a review, see Kerwin, 2005; Suchman, Pajulo, DeCoste, \& Mayes, 2006).

Recent developments in the neuroscience of addiction and parenting suggest a significant overlap in the neural circuitry involved with chronic drug use and parenting (Rutherford, Williams, Moy, Mayes, \& Johns, 2011; Strathearn, 2011; Strathearn, Fonagy, Amico, \& Montague, 2009). Chronic drug use appears to co-opt the same dopaminergic neural pathways recruited during caregiving, decreasing reward sensitivity, heightening stress activation, and potentially increasing vulnerability to relapse during caregiving activities.

Heroin and opioids are being increasingly used at younger ages more broadly across socioeconomic strata (Jones, 2008; Seelye, 2015). Moreover, increasing purity of available heroin has led to alarming rates of overdose (Buckley, 2009). 
As they move into childbearing years, substance users are at greater risk for experiencing diminished reward and heightened distress as they transition to parenthood.

Taken together, these findings suggest the need for parenting interventions provided during addiction treatment that target the emotional consequences of a hijacked stress-reward system, especially those related to the parenting role. Supporting the parent's emerging skills and capacities to manage challenging emotional experiences of parenthood concomitantly with addiction treatment may be a critical first step toward better parenting and may also promote relapse prevention. Moreover, understanding how addiction severity interacts with response to parenting interventions is also critical because it can help clarify which parenting interventions are the best match for parents with greater addiction severity.

\section{The Mothering From the Inside Out (MIO) Intervention}

MIO is a manualized 12-session individual therapy developed to enhance a mother's capacity for mentalization or reflective functioning $(\mathrm{RF})$ in the parenting role (Suchman \& Bers, 2015). RF refers to the capacity to recognize and make sense of mental (especially emotional) states, that is, how they influence behavior and their possible impact on relationships (Fonagy et al., 1995). MIO is based on the mentalization-based therapy model developed by Allen, Fonagy, and Bateman (2008), which emphasizes the restoration of mentalizing capacities under conditions of arousal by engaging in explicit, guided mentalization practice (Bateman \& Fonagy, 2012).

Parental RF refers to a parent's capacity to make sense of her own mental and emotional experiences in the parenting role and to make sense of the mental and emotional states that drive her child's behavior. Parental RF has many immediate and practical benefits. It enables a parent to make sense of a young child's mental and emotional experiences, which in turn may help the parent respond sensitively to the infant's emotional and physical needs (Slade, 2005). It also enables the parent to recognize, understand, and manage her own mental and emotional experiences and their impact on the child and caregiving relationship. This latter capacity is particularly relevant to mothers in addiction treatment because heightened distress and limited coping skills make them more vulnerable to emotional dysregulation and relapse.

MIO explicitly targets parental RF so that mothers can better manage emotional distress in the absence of neural reward that is common during chronic substance use episodes and early recovery from addiction. The short-term goals of MIO are to (a) provide a positive experience in a therapeutic relationship in which the mother's thoughts and emotions are taken seriously so that she can feel supported and understood and (b) begin a process of helping the mother make sense of her own and her child's underlying affective experiences and think about how these experiences are related to individual need, behavior, and development. The long-term goals of
MIO are to (a) support the mother's developing capacity for emotional regulation, (b) restore the mother's own capacity to engage in human attachment (e.g., replace attachment to a substance with attachment to the child), and (c) promote the mother's capacity to engage with and enjoy her child, tolerate her child's emotional distress, understand her child's emotional needs, and support her child's developing regulatory capacities.

During each MIO session, the mother determines the focus of the discussion. If the child is not the immediate topic, the therapist will bring the child into mind when timing seems appropriate. Stressful situations, particularly those where the mother's capacity for RF is challenged, are considered in detail. The therapist invites the mother to reengage in the process of RF; that is, to consider thoughts, feelings, and intentions in herself, her child, and others as the therapist and parent review the stressful situation together. The therapist is careful not to shift the focus to the child too early. Often, the focus begins with recognizing the mother's own affective distress and understanding its mental and emotional antecedents. In this way, MIO emphasizes engagement in a mentalizing process rather than specifying a particular content. The therapist provides relevant developmental guidance when the mother's expectations for the child appear to be unrealistic. The therapist also suggests parenting strategies that are likely to promote secure attachment. The therapist's curious, inquisitive, not-knowing stance is considered essential to the therapeutic process because of the opaque and transient nature of mental states and to encourage the mother to remain actively engaged in a mentalizing process (for further details about the MIO intervention, see Suchman, DeCoste, Ordway, \& Bers, 2013). In this study, MIO was delivered by two PhD-level clinical psychologists (including N.S.).

\section{First Randomized Trial}

We previously reported findings from the first randomized, controlled trial with 47 mothers enrolled in substance abuse treatment and caring for a child between birth and 3 years of age. In this trial, we tested MIO in comparison with parent education (PE), a 12-session individual psychoeducational comparison intervention where developmental guidance and parenting strategies were provided by an individual counselor (see Suchman, DeCoste, McMahon, Rounsaville, \& Mayes, 2011). At the end of 12 sessions, in comparison with mothers who received PE, those who received MIO demonstrated higher levels of RF, representational coherence, and caregiving behavior, and these differences were sustained at a 6-week follow-up. At the 6-week follow-up, both groups also had significantly reduced psychiatric symptoms and substance use. MIO children showed clearer communication bids with their mother at posttreatment, and these differences were also sustained at the 6-week follow-up. A test of treatment mechanisms (see Suchman, DeCoste, Rosenberger, \& McMahon, 2012) showed that improvement in maternal RF capacity 
was directly related to improvement in maternal caregiving behavior.

\section{Current Investigation}

Here we report findings from the second randomized, controlled trial testing MIO in comparison with PE in a sample of 87 mothers enrolled in substance abuse treatment and caring for a child between 11 months and 5 years of age. The aims of this second trial, in addition to replicating the first trial in a larger sample, were to (a) determine if results would be replicated in a sample of mothers caring for children in a wider age range (up to 5 years old), (b) measure treatment impact on child attachment, (c) examine treatment outcomes at 3-month and 12-month follow-up, and (d) explore potential moderating effects of addiction severity.

We predicted that, in comparison with mothers enrolled in $\mathrm{PE}$, mothers enrolled in MIO would demonstrate (a) greater capacity for mentalizing and more coherent caregiving representations at the end of treatment with group differences sustained at the 3-month follow-up, (b) more sensitive caregiving behavior by the end of treatment with group differences sustained at the 3-month and 12-month follow-up visits, and (c) lower levels of relapse to substance use and lower levels of psychiatric distress at the 3-month follow-up.

We also predicted that, in comparison with children of mothers enrolled in PE, children with mothers enrolled in MIO would demonstrate (a) more secure attachment at posttreatment, and (b) better communication, involvement, and dyadic reciprocity with their mothers at the end of treatment, with group differences sustained at the 3-month and 12month follow-up visits.

With regard to addiction severity, we expected that MIO would confer a protective benefit to mothers and children. Specifically, for mothers with greater addiction severity, MIO would demonstrate a greater protective function than PE for levels of maternal RF, quality of mother-child interactions, and child attachment security.

\section{Method}

\section{Overview}

This trial was conducted on site at a substance abuse treatment center located in a small northeastern city where many of its clients are exposed to urban problems (e.g., crime, poverty, and minimal affordable housing) typically identified with larger cities. Interested mothers enrolled in outpatient services at the treatment center who were caring for a child between 11 and 60 months of age and eligible to participate were randomized to 12 sessions of a manualized intervention: MIO or 12 sessions of PE, a manualized, individual, psychoeducational active control comparison developed for this study. The study included a 6-week baseline assessment, a 12-week intervention, and a 3-month and 12-month followup assessment. Treatment fidelity was measured using a scale developed during the first randomized trial. Treatment outcomes included maternal RF, representational coherence, maternal psychiatric symptoms, maternal substance abuse, mother-child interaction quality, and child attachment status. All procedures were approved by the Yale University School of Medicine Institutional Review Board.

\section{Recruitment and consent procedures}

Mother-child dyads were eligible to participate if the mother was enrolled in the outpatient treatment program for her substance abuse, English speaking, and caring for a child between 11 and 60 months of age. Dyads were excluded if the mother had severe mental health problems (e.g., suicidality and psychosis), significant cognitive impairment, or required inpatient hospitalization or detoxification. Dyads were also excluded if the target child had a serious illness or significant developmental delay.

Mothers were recruited via clinician referrals, research assistant visits to clinic medication lines and group meetings, research interest forms, flyers posted throughout the treatment clinic, and word of mouth. Interested mothers were screened for eligibility by research assistants either in person or by phone. Mothers who met eligibility criteria met individually with a research assistant to complete informed consent procedures. Mothers caring for more than one eligible child were allowed to choose which child would participate with them. Mothers who had a child living with a relative were permitted to enroll if they had regular contact with the child (e.g., 3-4 days per week). In this case, the relative or legal guardian of the child provided consent for the child's participation. During the informed consent meeting, permission was asked to access program clinical records to ascertain information about attendance and relapses to substance use. Limits of confidentiality and the protection of research records under a Certificate of Confidentiality were explained. The compensation schedule for research assessments was also reviewed.

\section{Sample}

Mothers. One hundred mothers caring for a child between 11 and 60 months of age consented to participate. Of these mothers, 87 completed the initial intake evaluation and were randomized to treatment (40 MIO, $47 \mathrm{PE})$, constituting the intention to treat sample $(n=87)$. On average, mothers were $29.68(S D=5.37)$ years old, had completed 12.39 $(S D=2.16)$ years of education, and were caring for 1.67 $(S D=0.97)$ children under 16 years of age. Mothers' verbal intelligence (mean standardized score $=91.48, S D=10.35$ ) and nonverbal intelligence (mean standardized score $=$ 97.47, $S D=12.87$ ) fell within the range considered normal. Most of the mothers were Caucasian (77\%), $13.8 \%$ were African American, 3.4\% were Hispanic or Latino, and $5.7 \%$ were of mixed race. A large percentage of the mothers had never been married $(42.5 \%), 34.5 \%$ were cohabitating with a partner, $13.8 \%$ were married, $6.9 \%$ were divorced, 
and $2.3 \%$ were separated. A majority $(69.8 \%)$ of the mothers were living independently, $35.6 \%$ depended on family or friends for housing, and $4.6 \%$ were homeless at the time of enrollment. Approximately one-third (32.2\%) of children were involved with the Child Protective Services. Most mothers $(89 \%)$ carried a primary diagnosis of heroin or nonprescription opioid dependence, $6.1 \%$ met criteria for alcohol dependence; $3.7 \%$ were diagnosed with cocaine dependence, and $1.2 \%$ with cannabis dependence. Most $(72.4 \%)$ were enrolled in methadone-maintenance, and $12.6 \%$ were enrolled in suboxone treatment. A majority of mothers had significant family histories of substance abuse, including their own mother $(53.5 \%)$ and father $(75.9 \%)$ and the target child's father $(76.7 \%)$. On average, cannabis and alcohol use was initiated in early teens, and other drug use (e.g., heroin, opioids, cocaine, and hallucinogens) was initiated in later teens or early 20s. All mothers reported having experienced withdrawal and dependence symptoms at some point in their substance use history. On average, mothers reported clinically significant levels of psychiatric distress (Brief Symptom Index Global Severity Index mean $\mathrm{T}$ score $=60.37, S D=$ 10.06). As shown in Table 1 , the only significant group difference in baseline maternal characteristics involved cocaine use during pregnancy; more MIO than PE mothers reported use.

Fathers. Per mothers' reports, fathers of target children were 34.28 years old $(S D=7.48)$, on average, and $54.0 \%$ were living separately from the mother and target child. A majority of fathers were employed $(63.4 \%)$ but also had histories of substance use (76.7\%). As shown in Table 1, there were no significant group differences in paternal characteristics.

Target children. Pregnancy with the target child was generally unplanned (79.7\%), and mothers reported learning about their pregnancy and attending their first prenatal visit at week 7.34 ( $S D=4.82)$. A majority of mothers $(96.6 \%)$ reported they continued to receive prenatal care throughout their pregnancy. A majority of mothers $(83.1 \%)$ reported using nicotine during pregnancy, $25 \%$ reported using cocaine, $16.9 \%$ reported using heroin, $15.3 \%$ reported using cannabis, $10.2 \%$ reported using other opioids, $10.2 \%$ reported using alcohol, and $1.7 \%$ reported using hallucinogens. The average birth weight of target children was $6.96 \mathrm{lb}(S D=1.46)$. More than three-quarters $(77.8 \%)$ of the target children whose mothers were enrolled in methadone maintenance required a methadone detox at birth, and $37.5 \%$ of the children whose mothers were prescribed suboxone treatment required a suboxone detox. Many mothers and infants (48.1\%) were separated at birth, and infants remained in the hospital for an average of 14.38 days $(S D=11.39$ ). All mothers who were separated reported visiting their infants in the hospital; $30 \%$ reported visiting daily, $30 \%$ reported visiting nightly, $35 \%$ reported visiting every day and night, and $5 \%$ reported visiting some days and nights. Almost all mothers (98.3\%) reported that their infants had an assigned pediatrician, and the average age of first pediatric visit was 2.55 months $(S D=2.62)$. At baseline, $28.8 \%$ of mothers expressed developmental concerns about the target child.

Target children were 27.62 months of age $(S D=14.73)$, on average, and $54 \%$ were male. A large majority $(95.4 \%)$ lived with their mother at baseline whereas $4.6 \%$ lived with another family member and had frequent visits with the mother (e.g., 3-4 days per week). On the Bayley Developmental Screen Cognitive Scale, $12.5 \%$ of children between 11 and 26 months of age scored as emerging risk, and $2.5 \%$ scored at risk. On the Bayley Receptive Communication Scale, $18.9 \%$ scored as emerging risk and 5.4\% scored at risk. On the Bayley Expressive Communication screen, 16.2\% scored as emerging risk and $0 \%$ scored at risk. On the Early Screening Profile Cognitive/Language Scale, 5.3\% of children between 37 and 60 months scored below average. As shown in Table 1, there were no significant group differences in target child characteristics.

\section{Assessment procedures}

Baseline. Following consent, the mother and participating child were scheduled for six weekly baseline assessment visits (1- to 2-hr duration) during which the mother completed a battery of assessments including an initial intake interview as well as measures of RF, mental representations of caregiving, psychiatric symptoms, substance use, and intelligence (see Table 2 for assessment schedule and Table 3 for baseline scores). She and her child also completed assessments measuring interaction quality, child developmental status, and child attachment status. Mothers received compensation, and children received a developmentally appropriate toy for each completed assessment. Immediately following the intake interview, in the second baseline week, mothers were randomized to treatment and introduced to their assigned MIO therapist or PE specialist. Early randomization was considered important for building a working alliance, preventing attrition, and processing any emotional distress triggered by the assessments.

Treatment. During the 12-week treatment phase, mothers met weekly for a 1-hr individual session with their assigned MIO therapist or PE specialist and completed brief surveys about their recent substance use and additional services received in the community. Brief psychiatric symptom questionnaires were completed every 4 weeks.

Posttreatment. At the end of the 12 treatment sessions, mothers completed 4 posttreatment visits (1- to 2 -hr duration) during which maternal measures of RF, mental representations of caregiving, psychiatric symptoms, and substance use were repeated. Mothers and target children also repeated assessments of interaction quality and child attachment status.

Follow-up. During the first 3 months following the posttreatment assessments, mothers were scheduled for brief (30 min) 
Table 1. Baseline characteristics of mothers, fathers, and target children

\begin{tabular}{|c|c|c|c|c|}
\hline & & & $t / \chi^{2}$ & $d^{a} / \phi$ or $V^{b}$ \\
\hline Maternal demographic factors & $\mathrm{MIO}(n=40)$ & $\mathrm{PE}(n=47)$ & & \\
\hline Age & $29.89(5.10)$ & $29.43(5.73)$ & 0.40 & 0.06 \\
\hline Education (years) & $12.10(1.87)$ & $12.64(2.38)$ & 1.16 & 0.17 \\
\hline Unemployed & $75.00 \%$ & $85.10 \%$ & 1.40 & 0.13 \\
\hline Ethnicity & & & 0.44 & 0.07 \\
\hline Caucasian & $80.00 \%$ & $74.50 \%$ & & \\
\hline Hispanic/Latino & $2.50 \%$ & $4.30 \%$ & & \\
\hline African American & $12.50 \%$ & $14.90 \%$ & & \\
\hline Other & $5.00 \%$ & $6.40 \%$ & & \\
\hline \multicolumn{5}{|l|}{ Intelligence } \\
\hline Standardized Verbal & $91.60(11.84)$ & $91.39(9.25)$ & -0.07 & 0.01 \\
\hline Standardized Non-Verbal & $95.04(14.50)$ & $99.30(11.37)$ & 1.26 & 0.23 \\
\hline Marital status & & & 2.23 & 0.16 \\
\hline Never married & $50.00 \%$ & $36.20 \%$ & & \\
\hline Cohabitating & $32.50 \%$ & $36.20 \%$ & & \\
\hline Divorced & $5.00 \%$ & $8.50 \%$ & & \\
\hline Separated & $2.50 \%$ & $2.10 \%$ & & \\
\hline Married & $10.00 \%$ & $17.00 \%$ & & \\
\hline Domicile & & & 3.03 & 0.19 \\
\hline Independent & $50.00 \%$ & $68.10 \%$ & & \\
\hline Dependent ${ }^{\mathrm{c}}$ & $45.00 \%$ & $27.70 \%$ & & \\
\hline Homeless & $5.00 \%$ & $4.30 \%$ & & \\
\hline Biological children & $1.90(1.15)$ & $2.04(1.08)$ & 0.60 & 0.09 \\
\hline DCF involved (current) & $35.00 \%$ & $29.80 \%$ & 0.27 & 0.06 \\
\hline \multicolumn{5}{|c|}{ Substance Use History } \\
\hline Primary diagnosis & & & $8.28 \dagger$ & 0.31 \\
\hline Heroin/opioids & $89.5 \%$ & $83.0 \%$ & & \\
\hline Alcohol & $2.6 \%$ & $8.5 \%$ & & \\
\hline Cocaine & $0.0 \%$ & $6.4 \%$ & & \\
\hline Cannabis & $0.0 \%$ & $2.1 \%$ & & \\
\hline PCP & $7.9 \%$ & $0.0 \%$ & & \\
\hline \multicolumn{5}{|l|}{ Opiate replacement therapy } \\
\hline Methadone & $74.5 \%$ & $70.0 \%$ & 0.22 & 0.05 \\
\hline Suboxone & $15.0 \%$ & $10.6 \%$ & 0.37 & 0.07 \\
\hline Addiction severity & $9.95(2.36)$ & $10.38(2.25)$ & -0.63 & \\
\hline \multicolumn{5}{|l|}{ Family history of substance abuse } \\
\hline Own mother & $59.0 \%$ & $48.9 \%$ & 0.86 & 0.10 \\
\hline Own father & $76.3 \%$ & $74.4 \%$ & 0.04 & 0.02 \\
\hline Target child's father & $79.5 \%$ & $73.5 \%$ & 0.36 & 0.07 \\
\hline \multicolumn{5}{|l|}{ Early initiation } \\
\hline Alcohol $^{d}$ & $42.5 \%$ & $42.6 \%$ & 0.00 & 0.00 \\
\hline Cannabis $^{d}$ & $55.3 \%$ & $52.5 \%$ & 0.07 & 0.03 \\
\hline Heroin $^{e}$ & $47.5 \%$ & $51.1 \%$ & 0.11 & 0.04 \\
\hline Opioids ${ }^{e}$ & $67.5 \%$ & $70.2 \%$ & 0.07 & 0.03 \\
\hline Cocaine $e^{e}$ & $72.5 \%$ & $55.3 \%$ & 2.74 & 0.18 \\
\hline Withdrawal symptoms & $100.0 \%$ & $100.0 \%$ & NA & NA \\
\hline Dependence symptoms & $100.0 \%$ & $97.9 \%$ & 0.86 & 0.10 \\
\hline Psychiatric distress $(T \geq 60)$ & 65.0 & 57.4 & 0.52 & 0.08 \\
\hline Moderate depression $(\mathrm{BDI} \geq 14)$ & 62.5 & 51.1 & 1.15 & 0.12 \\
\hline \multicolumn{5}{|c|}{ Target Child's Father Characteristics } \\
\hline Age & $34.30(8.02)$ & $34.26(7.14)$ & -0.02 & 0.00 \\
\hline Living at home & 42.5 & 48.9 & 0.36 & 0.06 \\
\hline Employed & 60.9 & 66.7 & 0.15 & 0.06 \\
\hline History of substance use & 73.5 & 79.5 & 0.36 & 0.07 \\
\hline
\end{tabular}


Table 1 (cont.)

\begin{tabular}{|c|c|c|c|c|}
\hline & & & $t / \chi^{2}$ & $d^{a} /$ p or $V^{b}$ \\
\hline \multicolumn{5}{|c|}{ Pre- and Postnatal History (With Target Child) } \\
\hline \multicolumn{5}{|l|}{ Substances used during pregnancy } \\
\hline Cigarettes & 84.2 & 76.1 & 0.85 & 0.10 \\
\hline Cocaine & 28.9 & 8.7 & $5.82 *$ & 0.26 \\
\hline Heroin & 17.9 & 15.2 & 0.11 & 0.04 \\
\hline Opioids & 20.5 & 6.5 & $3.67 \dagger$ & 0.21 \\
\hline Cannabis & 15.8 & 10.9 & 0.44 & 0.07 \\
\hline Alcohol & 10.5 & 6.5 & 0.44 & 0.07 \\
\hline Hallucinogens & 5.3 & 1.0 & 2.48 & 0.17 \\
\hline Infant birth weight & $6.73(1.48)$ & $7.16(1.44)$ & 1.13 & 0.21 \\
\hline \multicolumn{5}{|l|}{ Infants requiring detox at birth } \\
\hline Methadone detox $f$ & 84.2 & 68.0 & 1.51 & 0.19 \\
\hline Suboxone detox ${ }^{g}$ & 37.5 & 33.3 & 0.04 & 0.03 \\
\hline Days hospitalized & $16.61(15.24)$ & 12.37 (11.98) & -1.19 & 0.04 \\
\hline Age at pediatric visit (months) & $2.92(3.01)$ & $2.21(2.23)$ & -1.01 & 0.22 \\
\hline \multicolumn{5}{|c|}{ Target Child Characteristics } \\
\hline Age (months) & $27.83(15.75)$ & $27.45(13.97)$ & -0.12 & 0.02 \\
\hline Male & 52.5 & 55.3 & 0.07 & 0.03 \\
\hline Lives with & & & 0.03 & 0.02 \\
\hline Mother & 95.0 & 95.7 & & \\
\hline Another relative & 5.0 & 4.3 & & \\
\hline \multicolumn{5}{|c|}{ Developmental Assessment } \\
\hline \multicolumn{5}{|l|}{ Bayley (11-36 months) $n=40^{c}$} \\
\hline Cognitive & & & 1.32 & 0.18 \\
\hline Competent & 80 & 90 & & \\
\hline Emerging risk & 15 & 10 & & \\
\hline At risk & 5 & 0 & & \\
\hline Receptive communication & & & 2.26 & 0.25 \\
\hline Competent & 72.2 & 78.9 & & \\
\hline Emerging risk & 16.7 & 21.1 & & \\
\hline At risk & 11.1 & 0.0 & & \\
\hline Expressive communication & & & 0.01 & 0.01 \\
\hline Competent & 83.3 & 84.2 & & \\
\hline Emerging risk & 16.7 & 15.8 & & \\
\hline At risk & 00.0 & 00.0 & & \\
\hline \multicolumn{5}{|c|}{ Early Screening Profile (37-60 months) $n=19$} \\
\hline Cognitive/language & & & 0.78 & 0.20 \\
\hline Above average & 37.5 & 36.4 & & \\
\hline Average & 62.5 & 54.5 & & \\
\hline Below average & 0.0 & 9.1 & & \\
\hline
\end{tabular}

${ }^{a}$ Effect size $d: 0.20=$ small, $0.50=$ medium, $0.80=$ large $($ Cohen, 1988).

${ }^{b}$ Cramer $\phi$ was used for two categories and Cramer $V$ was used for $>2$ categories, $0.10=$ small, $0.30=$ medium, $0.50=$ large.

${ }^{c}$ Living in supervised housing or in the home of a family member.

${ }^{d} \leq 13$ years of age.

$e^{e} \leq 18$ years of age.

${ }^{f}$ Children of methadone-maintained mothers only.

${ }^{g}$ Children of suboxone-prescribed mothers only.

$\dagger p<.10 .{ }^{*} p<.05$ (two tailed).

twice monthly visits to complete assessments of psychiatric symptoms, substance use, and additional services received. Then, during follow-up weeks 12 through 15, maternal measures of RF, mental representations of caregiving, psychiatric symptoms, and substance use were repeated. Mothers and target children also repeated assessments of interaction quality. On the 1-year anniversary of study completion, mothers returned with the target child for a follow-up visit to repeat 
Table 2. Assessment schedule

\begin{tabular}{|c|c|c|c|c|c|c|}
\hline Measure & BL & Weekly & Monthly & Post & FU1 & FU2 \\
\hline Intake Evaluation & $\mathrm{x}$ & & & & & \\
\hline Kaufman Brief Intelligence Test & $\mathrm{x}$ & & & & & \\
\hline 11-36 months Bayley Scales of Infant and & & & & & & \\
\hline Toddler Development Screening Test & $\mathrm{x}$ & & & & & \\
\hline 37-60 months Early Screening Profiles & $\mathrm{x}$ & & & & & \\
\hline Parent Development Interview & $\mathrm{x}$ & & & $\mathrm{x}$ & $\mathrm{x}$ & \\
\hline Working Model of the Child Interview & $\mathrm{x}$ & & & $\mathrm{x}$ & $\mathrm{x}$ & \\
\hline Curiosity Box Paradigm & $\mathrm{x}$ & & & $\mathrm{x}$ & $\mathrm{x}$ & $\mathrm{x}$ \\
\hline Strange Situation Paradigm & $\mathrm{x}$ & & & $\mathrm{x}$ & & \\
\hline Beck Depression Inventory & & & $\mathrm{x}$ & & & \\
\hline Brief Symptom Inventory & & & $\mathrm{x}$ & & & \\
\hline Timeline Follow Back Interview & & $\mathrm{x}$ & & & & \\
\hline Weekly Checklist of Services & & $\mathrm{x}$ & & & & \\
\hline
\end{tabular}

one assessment of interaction quality, and mothers were also interviewed about any changes they experienced in the areas of parenting, education, employment, housing, and legal involvement (mother-child interaction findings are reported here; follow-up interview results are forthcoming).

Child care. Developmentally informed child care was available to all target children in the study at every visit. Research staff received weekly supervision from the developmental specialist to discuss concerns about child safety and development. On average, $\mathrm{MIO}$ children received $49.96 \mathrm{hr}(S D=$ 23.30), and PE children received $41.04 \mathrm{hr}(S D=23.72)$ of child care per study month, and group differences were not significant $(t=1.76, p=.08, d=0.27)$.

Compensation and attendance incentives. Procedures used to minimize potential barriers to participation (e.g., providing a child-friendly environment with developmentally informed child care, offering healthy snacks, diapers, spare clothing, and bus passes, providing graduate certificates upon completion) are described in detail elsewhere (see Suchman et al., 2013). Economic compensation for research visits were also structured to maximize motivation for assessment completion. Payment for assessment completion increased incrementally from baseline ( $\$ 10-\$ 15$ per assessment) through follow-up (\$35-\$40 per assessment). Bonus payments of $\$ 5$ to $\$ 10$ were also provided for on time assessment completion. Target children received a small developmentally appropriate toy for their participation in all assessments.

Attendance. Of the 87 randomized mothers, 70 (80\%) completed baseline assessments and continued to treatment. Reasons for attrition included transportation problems, relocation, family issues, substance use relapse, illness, and discharge or withdrawal from the addiction program. On average, MIO mothers attended $71 \%$ of their clinical appoint-

Table 3. Baseline scores for parenting and child outcome measures

\begin{tabular}{|c|c|c|c|c|}
\hline Maternal Reflective Functioning & MIO & PE & $F$ & $d$ \\
\hline Mean RF & $3.08(0.50)$ & $3.12(0.49)$ & 0.38 & 0.06 \\
\hline Potential RF & $4.39(0.90)$ & $4.57(0.73)$ & 0.89 & 0.16 \\
\hline \multicolumn{5}{|l|}{ Maternal working model of the child } \\
\hline Overall coherence & $2.47(0.31)$ & $2.60(0.40)$ & 1.54 & 0.26 \\
\hline \multicolumn{5}{|l|}{ Psychiatric symptoms } \\
\hline BDI depression score & $15.78(10.21)$ & $15.04(11.92)$ & -0.30 & 0.05 \\
\hline BSI global symptom (T score) & $60.38(10.10)$ & $60.36(10.14)$ & -0.01 & 0.00 \\
\hline \multicolumn{5}{|l|}{ Mother-child dyadic adjustment } \\
\hline \multicolumn{5}{|l|}{ Curiosity box } \\
\hline Maternal sensitivity & $3.55(0.70)$ & $3.47(0.77)$ & -0.50 & 0.08 \\
\hline Child involvement & $3.41(0.63)$ & $3.40(0.64)$ & -0.06 & 0.01 \\
\hline Dyadic reciprocity & $3.58(0.94)$ & $3.38(1.03)$ & -0.93 & 0.14 \\
\hline \multicolumn{5}{|l|}{ Child attachment } \\
\hline Strange Situation & & & 3.45 & 0.24 \\
\hline Secure & 57.1 & 58.1 & & \\
\hline Insecure & 21.4 & 35.5 & & \\
\hline Disorganized & 21.4 & 6.5 & & \\
\hline
\end{tabular}


ments, and $81 \%$ of their research appointments, whereas PE mothers attended $75 \%$ of their clinical appointments and $84 \%$ of their research appointments. There were no significant group differences in clinical $(t=1.18, p=.24, d=$ $0.06)$ or research $(t=1.75, p=.08, d=0.06)$ attendance.

\section{PE comparison}

PE was designed and manualized (DeCoste, Dalton, de las Heras Kuhn, \& Dennehy, 2010) to represent psychoeducational parenting programs that are typically available in community settings. PE was designed as an individual intervention tailored to the specific interests and concerns of the parent in order to control for treatment dose and the opportunity to form a working alliance. PE is a structured intervention that provides developmental guidance and parenting strategies for challenges that are typically encountered by parents with young children (e.g., child tantrums, bed wetting, sleep habits, limit setting, and developmental milestones) and challenges that are typical for parents in substance abuse treatment (e.g., keeping children safe and self-care). Mothers met weekly with their assigned PE specialist to review a pamphlet chosen by the mother. Pamphlets were written at a fourth-grade reading level.

\section{Constructs and measures}

Treatment integrity. The Revised MIO/PE Adherence Rating Scale (Suchman, Rosenberger, \& DeCoste, 2010) was used to measure treatment integrity. The scale contains generic items measuring alliance-building efforts that were expected to occur equally in both $\mathrm{MIO}$ and $\mathrm{PE}$ and unique $M I O$ and $P E$ items measuring behaviors that were expected to occur primarily in $\mathrm{MIO}$ or $\mathrm{PE}$, respectively. To streamline rating procedures and insure that items were robust, the original 23-item scale (Suchman, Rosenberger, \& DeCoste, 2006) was reduced to 18 items by combining those that overlapped. Next, three independent raters trained by author N.S. rated 36 randomly selected sessions to establish interrater reliability. Interclass correlations for 7 of the 18 items were considered inadequate $(<0.70)$, and these items were omitted. Interclass correlations for the remaining 11 items comprising the final scale ranged from $0.74(p<.05)$ to $0.96(p<.001)$. Raters coded 428 sessions from a randomly selected pool of 37 subjects $(\mathrm{MIO}=15$ and $\mathrm{PE}=22)$ who attended 11 sessions, on average.

Total scale scores were computed by summing items on each subscale scale. For each session, a score of $>3$ on the generic scale (range $=0-6$ ) is considered adequate. For MIO sessions, a score of $>2$ on the mentalizing for child and relationship subscale (range $=0-6$ ) and a score of $>3$ on the mentalizing for self subscale (range $=0-6$ ) are considered adequate fidelity. For PE sessions, a score of $>2$ on the unique $P E$ scale (range $=0-4$ ) is considered adequate. As shown in Table 4, MIO and PE clinicians achieved adequate fidelity to their respective interventions.
To confirm intervention construct validity, a principal components analysis was conducted first, extracting eigenvalues greater than 1. A screeplot indicated a five-factor solution as the best fit. Next, a Varimax rotation was used in a second factor analysis with extraction restrained to five factors. As shown in Table 4, five robust factors were identified with two factors representing the MIO intervention, one factor representing the $\mathrm{PE}$ intervention, and two factors representing generic interventions.

Discriminant validity was confirmed using independent $t$ tests, and results are reported in Table 4. MIO clinicians scored significantly higher than PE clinicians on both unique $M I O$ subscales, and PE clinicians likewise scored significantly higher than MIO clinicians on the unique PE scale. $\mathrm{MIO}$ and PE clinicians were not expected to differ in fidelity to generic interventions, and this absence of difference is reflected in the nonsignificant $t$ test results.

Intake interview. A 1.5-hr structured clinical interview was used to gather information about the mother's developmental and family history; substance use (including during pregnancy); psychiatric and trauma history; medical, legal, and employment history; and pre- and postnatal history of the target child. Information from this interview was used to establish primary substance use and psychiatric diagnoses, determine addiction severity, and identify baseline demographic and psychosocial characteristics of the sample.

Maternal addiction severity. A 15-item binary scale representing cumulative risk for severe addiction was computed to serve as a moderator, using items from the intake evaluation and psychiatric survey. Scale item domains included (a) family history of substance abuse and mental illness, (b) early onset of substance use initiation (e.g., alcohol and cannabis before age 13; heroin and cocaine use before age 18), (c) higher than average endorsement of triggers and withdrawal and dependence symptoms, (d) higher than average endorsement of exposure to traumatic events, and (e) endorsement of clinically significant levels of psychiatric distress. The scale sum (range $=0-15$ ) was used to represent overall addiction severity.

Maternal intelligence. The Kaufman Brief Intelligence Test (Kaufman \& Kaufman, 1990) was used to characterize the sample in terms of IQ. The Kaufman Brief Intelligence Test is a brief (30 min) standardized intelligence screening measure with good reliability and concurrence with other widely used intelligence measures (Miller, 1995; Young, 1995).

Maternal representations. Maternal RF and internal working model of the child were the two representational constructs measured.

Maternal RF. The 33-item Parent Development Interview (PDI; Slade, Aber, Berger, Bresgi, \& Kaplan, 2003) was used to measure parental RF. The PDI is a semistructured inter- 
Table 4. MIO and PE Fidelity Scale item factor loadings and mean comparisons

\begin{tabular}{|c|c|c|c|c|c|c|c|c|}
\hline \multirow[b]{2}{*}{ Scale Item } & \multicolumn{5}{|c|}{ Factor Loadings } & \multicolumn{2}{|c|}{ Mean $(S D)$} & \multirow[b]{2}{*}{$t$} \\
\hline & 1 & 2 & 3 & 4 & 5 & MIO & $\mathrm{PE}$ & \\
\hline \multicolumn{9}{|l|}{ Unique to $\mathrm{MIO}$} \\
\hline Mentalizing for child and relationship & & & & & & $2.06(0.84)$ & $1.48(0.61)$ & $2.42 *$ \\
\hline $\begin{array}{l}\text { 1. Developmental guidance about child's } \\
\text { emotional needs and attachment-based } \\
\text { parenting strategies }\end{array}$ & .12 & .79 & .43 & .20 & -.08 & & & \\
\hline $\begin{array}{l}\text { 2. Explores thoughts and emotions underlying the } \\
\text { child's behavior }\end{array}$ & .44 & .79 & .13 & .09 & .03 & & & \\
\hline $\begin{array}{l}\text { 3. Explores mother's mental representations of } \\
\text { her child and their relationship }\end{array}$ & .32 & .76 & .13 & -.06 & .07 & & & \\
\hline Mentalizing for self & & & & & & $3.48(1.49)$ & $0.86(0.66)$ & $6.38 * * * a$ \\
\hline $\begin{array}{l}\text { 4. Explores thoughts and emotions underlying } \\
\text { mother's behaviors }\end{array}$ & .92 & .21 & .07 & .06 & .08 & & & \\
\hline $\begin{array}{l}\text { 5. Helps mother make sense of her own thoughts } \\
\text { and emotions }\end{array}$ & .90 & .19 & -.10 & .14 & -.10 & & & \\
\hline $\begin{array}{l}\text { 6. Explores how mother's thoughts and emotions } \\
\text { might affect the child }\end{array}$ & .79 & .46 & .13 & -.00 & -.03 & & & \\
\hline Unique to $\mathrm{PE}$ & & & & & & $1.63(0.64)$ & $2.24(0.90)$ & $-2.40 *$ \\
\hline $\begin{array}{l}\text { 7. Developmental guidance about child behavior } \\
\text { and behavior-based parenting strategies. }\end{array}$ & .02 & .20 & .97 & -.00 & -.03 & & & \\
\hline $\begin{array}{l}\text { 8. Developmental guidance about child's } \\
\text { physical safety. }\end{array}$ & .03 & .21 & .97 & .05 & -.04 & & & \\
\hline Generic & & & & & & $4.00(0.06)$ & $3.98(0.14)$ & 0.49 \\
\hline $\begin{array}{l}\text { 9. Builds alliance through listening and making } \\
\text { encouraging, supportive statements }\end{array}$ & .12 & .08 & .15 & .86 & .14 & & & \\
\hline $\begin{array}{l}\text { 10. Remains neutral during descriptions reflecting } \\
\text { questionable parenting attitudes and strategies. }\end{array}$ & .04 & .03 & -.08 & .89 & .11 & & & \\
\hline $\begin{array}{l}\text { 11. Assists mother to address concrete, practical } \\
\text { problems or immediate crises when asked. }\end{array}$ & -.03 & .03 & -.07 & .25 & .96 & & & \\
\hline
\end{tabular}

${ }^{a}$ Equal variances not assumed.

$* p<.05$. ${ }^{* * *} p<.001$ (two tailed).

view that requires parents to describe specific interactions with the target child. Some questions (called "demand" questions) are designed to explicitly probe for mental states in the mother (e.g., "Have you ever been angry as a parent?" and "Can you tell me about a time recently when you felt that way?") or the child (e.g., "Does your child ever get emotionally upset?" and "Can you tell me about the last time that happened?") Other questions (called "permit" questions) that have a more general focus (e.g., "Can you tell me about a time in the last week that you and your child really clicked?") are designed to permit rather than demand mentalizing activity. For this study, a shorter, 14-item version of the PDI was developed, with permission from the measure's primary author, in order to minimize assessment burden, avoid overlap with the Working Model of the Child Interview, and include positive emotion items (e.g., "Have you ever felt deeply touched or moved as a parent?") because mothers with addiction problems sometimes have difficulty mentalizing about positive affect. Each question is followed by a probe about the mother's and the child's experience. This version typically required $1 \mathrm{hr}$ to administer.

Interviews were videorecorded and transcribed verbatim by a professional transcription service. Dates, ID numbers, and identifying information were masked to keep coders blind to subject and time point. Each PDI interview item is coded on a -1 to 9 scale depending on the amount and quality of apparent mentalizing. A score of $\leq 3$ is considered "prementalizing" because it represents the absence of awareness of mental states except for vague, unelaborated, cliché-like references (e.g., "He doesn't have a care in the world"). A score of 5 is considered the benchmark for adequate mentalizing and indicates a demonstrated awareness of mental states and how they influence behaviors and relationships (e.g., "I know when she likes her snack because she smiles and claps her hands . . . and that makes me proud"). Higher scores indicate more complex and nuanced understanding of the nature of mental states and how they influence behavior and relationships. All PDIs were coded by a colleague at another institution (author J.B.), who was trained by the PDI author and author N.S., and remained blind to all other information about subjects and treatment assignments. A randomly selected sample of 10 interviews was used to assess interrater reliability. Interclass correlations for 2 items were $<0.70$, and the items were therefore omitted. Interclass correlations for the remaining 12 items ranged from $0.77(p<.05)$ to $0.98(p<.001)$. The outcome score of greatest interest was 
the mean RF score for all 12 items, which yielded a 0.80 Cronbach $\alpha$ for this sample. We were also interested in examining the highest level of RF achieved on any single item as an indicator of each subject's potential for mentalizing (this variable was labeled potential RF).

Maternal Working Model of the Child. The Working Model of the Child Interview (Zeanah \& Benoit, 1993) is a semistructured interview used to assess the content and quality of a parent's mental representations of children ages birth to 6 . The interview includes questions about the mother's perceptions of distinctive characteristics of the child and their relationship, focusing primarily on times when the child's attachment needs are typically activated (e.g., parent-child separations, child illness, or injury). For this study, a 15item version of the interview was developed with permission from the measure's primary author to minimize assessment burden and avoid overlap with PDI items. Six 5-point items representing distinct representation characteristics were used to characterize representation quality. The items include richness (degree of detail and elaboration about child's personality), openness (acceptance and flexibility in expectations for the child over time), coherence (clarity and credibility of narrative), caregiving sensitivity (recognition and responsiveness to child's emotional distress), acceptance (acknowledgement of parental role and responsibility and child's dependence on parent for safety and care), and emotional involvement (expression of emotional investment in the child and caregiving relationship). A score of 3 on any subscale (range $=1-5$ ) is considered the "benchmark" score for adequate though unremarkable quality. Scores $\leq 2$ are considered to represent potential risk for the caregiving relationship and child. Interviews were videorecorded and then coded by a clinical psychologist (trained by the senior author, N.S.), who was also a clinical consultant to the research team. A randomly selected sample of 10 interviews was used to assess interrater reliability. Interclass correlations for the 6 items ranged from 0.77 ( $p<.01)$ to $0.91(p<.001)$. The outcome score of greatest interest for this study was the mean score for all 6 items, representing overall coherence, which yielded a 0.88 Cronbach $\alpha$ for this sample.

Maternal psychiatric symptoms. Psychiatric symptoms were assessed with the widely used Brief Symptom Index (Derogatis, 1993) Global Severity Index (Cronbach $\alpha=0.97$ for this sample).

Maternal substance use. Substance use was measured with the Timeline Followback interview (TLFB; Sobell \& Sobell, 1992, 1996), a widely used calendar method for assessing substance use. The TLFB uses a calendar to gather retrospective estimates of an individual's daily substance use over a specified period of time. The TLFB has demonstrated good temporal stability for alcohol and psychoactive substance use (for both general and specific drug classes). The TLFB has also demonstrated convergent and discriminant validity; percentage of days of substance use for different time intervals have had moderate to high correlations with other widely used measures of substance use severity, including urine assay results (Fals-Stewart, O-Farrell, Freitas, McFarlin, \& Rutigliano, 2000). In the event of a missed visit, the TLFB is designed to capture data from the missing time interval. For each month of the mother's participation in the study, she received a score of " 0 " if she reported no use of the substance and a score of " 1 " if she reported at least one relapse during that month

Child developmental screening. Two brief developmental assessments were used to screen for possible delays in target children's functioning. For children 1 to 36 months of age, the Bayley Scales of Infant and Toddler Development Screening Test (third edition; Bayley, 2006) was used to assess whether they were "on track" in their current cognitive and language functioning. For children between 36 and 60 months of age, the Early Screening Profiles (Harrison et al., 1990) was used to assess cognitive and language functioning. Research assistants were trained and supervised by a developmental consultant to conduct the screenings with target children during the baseline phase so that assessment referrals could be made promptly for children identified as being at risk.

Mother-child interactions. To examine the quality of mother-child interactions under conditions of mild uncertainty, we used the curiosity box paradigm developed by Mayes, Carter, and Stubbe (1993). During the curiosity box paradigm, the mother and child explore a box with 12 toys in two sequential 5-min episodes. The first episode involves familiar toys and is used to acclimate the dyad to the exercise, and the second episode involves unfamiliar toys chosen to elicit mild uncertainty in the child (e.g., a realistic rubber snake or plastic bug replica). All sessions were videorecorded, and second episodes were coded with Feldman's system for Coding Interactive Behavior (Feldman, 1998). The Coding Interactive Behavior uses a 5-point scale for rating parent, child, and dyadic behaviors that are likely to promote or inhibit emotional regulation in the child and dyad. Three composite scales, including the 12-item maternal sensitivity scale (Cronbach $\alpha=.93$ ), the 8-item child involvement scale (Cronbach $\alpha=0.84$ ), and the 3-item dyadic reciprocity scale (Cronbach $\alpha=0.97$ ) were used to assess interaction quality. All sessions were coded by a rater from Dr. Feldman's lab who was blind to treatment assignment, time, and other subject information. A randomly selected subsample of 10 interactions was also coded by a certified coder on our research team (author C.D.) to assess interrater reliability. Interclass correlations for the 23 items ranged from $0.77(p<.05)$ to $0.99(p<.001)$.

Child attachment classification. The Strange Situation Procedure (SSP; Ainsworth, Blehar, Waters, \& Wall, 1978) was used to assess child attachment classification. There is no sin- 
gle method for coding attachment classification that covers the age span from 11 to 60 months. However, a commonly accepted approach to measuring young children's response to attachment-based parenting interventions (see Hoffman, Marvin, Cooper, \& Powell, 2006; Solomon \& George, 2008; Toth, Rogosch, Manly, \& Cicchetti, 2006) in samples of children in this (and broader) age ranges has been to use the Ainsworth SSP (8 episodes, 3 with a stranger; Ainsworth et al., 1978) for children younger than 24 months and the MacArthur Preschool SSP (5 episodes, with no stranger; Cassidy, Marvin, \& MacArthur Attachment Working Group, 1992) for children older than 24 months (and up to 54 months) and examine changes in attachment status across three global domains: secure, insecure, and disorganized. Although practice effects are only likely to occur when the SSP is repeated over a very short term (e.g., 2-4 weeks; see Solomon \& George, 2008), we took steps to further minimize practice effects by conducting the SSP in a new setting with a new stranger unfamiliar to the child and mother for each visit. We also used objects available for play that the children had likely not seen before (e.g., novel toys, objects, and materials). The SSP was video recorded and then coded by two off-site consultants who were reliable on both methods. Ten randomly selected SSPs were used to assess interrater reliability for assessment of global attachment classification and the interclass correlation for this domain was $0.72(p<.05)$. In addition, when confidence in codes was questionable, the coders conferred to arrive at a common score. Because we were most interested in testing group differences in rates of improvement in attachment classification from baseline to postassessment visits, we created a binary outcome where 0 represents either (a) no pre- to posttreatment change from insecure or disorganized classification or (b) a decline to a more insecure classification (e.g., secure $\rightarrow$ insecure; disorganized or insecure $\rightarrow$ disorganized). A score of 1 represents either (a) maintenance of a secure classification or (b) change to a more secure classification (e.g., disorganized $\rightarrow$ insecure; secure or insecure $\rightarrow$ secure).

\section{Data analysis}

$M I O$ versus $P E$ comparisons. Because we were most interested in testing the efficacy of MIO versus PE after controlling for preexisting group differences (rather than examining within-subject change; see Gottman \& Rushe, 1993; Rogosa, 1995), analysis of variance was considered the best approach for examining outcomes in RF, mental representation coherence, and mother-child interaction at the end of the treatment and follow-up periods, respectively. A graphic representation of these outcomes across time indicated that a linear model from baseline to follow-up was not supported, precluding the use of hierarchical linear modeling. Baseline scores were included as covariates in all analyses, and child age and gender were also included as covariates in analyses involving mother-child interactions. A generalized linear modeling repeated measures approach was used to test for Group $\times$
Time slope differences in outcomes collected on a weekly (e.g., substance use) or monthly basis (e.g., psychiatric symptoms). A two-tailed significance test and examination of effect size $d(0.20=$ small, $0.50=$ medium, and $0.80=$ large effect; Cohen, 1988) were used to identify meaningful group differences. Chi square analysis along with a Fischer exact test and Cramer phi $(0.10=$ small, $0.30=$ medium, and $0.50=$ large effect; Cohen, 1988) were used to test for group differences in change in attachment status.

All outcome analyses were conducted with both the intention to treat $(n=87)$ and the treatment completer $(n=67)$ samples. There were no significant group differences (MIO vs. PE) in the number or pattern of missing values, and therefore, bias due to missingness was ruled out. In the first sample, for subjects who completed baseline assessments only, missing values for later time points were replaced with baseline values as a conservative estimate that assumed no improvement. For subjects who completed baseline and posttreatment assessments but missed follow-up assessments ( $\leq 10 \%$ of the intention to treat sample), missing values were replaced by the group mean. To insure that mean substitution did not significantly alter (i.e., reduce) variance (see Schafer \& Graham, 2002), equivalence of variances was confirmed across the three time points and no significant differences were found. Results for the two samples were then compared, and for all reported outcomes, patterns were similar across the two samples. Therefore, only results for the intention-to-treat sample are reported here. Because the sample size is moderate, we report effect sizes as well as significance test results.

Moderation analyses. Regression analysis was used to test for presence of significant Addiction Severity $\times$ Treatment interactions in relation to improvement in RF, maternal sensitivity, child involvement, dyadic reciprocity, and child attachment status. Because simple change scores calculated with continuous scores (i.e., subtracting the baseline score from the posttreatment) contain inherent bias due to baseline scores, we followed methods recommended by Cohen and Cohen (1983, pp. 413-425) to remove baseline score bias. (Again, we were most interested in examining efficacy in terms of treatment group differences after controlling for baseline scores.) For continuous outcomes, posttreatment and followup scores were regressed on baseline scores, and the residual variance (with baseline variance partialed out) was used to represent change. This method provides a reliable estimate of outcome that is not affected by correlations between baseline and outcome scores. Next, the Addiction Severity $\times$ Treatment interaction term was computed with centered addiction severity scores. Finally, for continuous dependent variables, treatment condition, addiction severity score, and the interaction term were entered simultaneously into a standard linear regression analysis. Interactions with small to moderate or larger effect sizes $\left(f^{2}>0.02\right.$; Cohen, 1988) were further probed using linear regression analysis of simple slopes and $t$ tests to examine interaction patterns. 
For the binary outcome (change in attachment status), we used the binary scores described above. In a binary logistic regression analysis, change in attachment classification served as the outcome variable, with binary score 0 representing no change in insecure attachment or deterioration to a more insecure attachment, and binary score 1 representing maintenance of or improvement toward a secure classification. Significant interactions were further probed with $\chi^{2}$ analyses to examine interaction patterns.

\section{Results}

\section{$M I O$ versus $P E$ comparisons}

Maternal RF and representations. As shown in Table 5, compared to PE mothers, MIO mothers had marginally higher mean RF scores at the end of treatment $(d=0.20)$ and significantly higher mean RF scores at the end of the 3-month follow-up period $(d=0.36)$. MIO mothers' potential RF was significantly higher at posttreatment $(d=0.34)$ and followup $(d=0.82)$ visits compared with PE mothers, with MIO mothers' potential RF scores approaching the benchmark of 5 that indicates adequate RF.

Table 5 reveals that MIO mothers had significantly higher overall coherence scores than PE mothers at posttreatment $(d=0.41)$ and 3-month follow-up $(d=0.54)$ with scores approaching the benchmark score of 3 , indicating absence of risk and adequate though unremarkable representation quality.

Maternal psychiatric symptoms. As shown in Table 6, after controlling for baseline scores, PE mothers $(d=-0.64)$ showed a marginally lower levels of psychiatric distress than MIO mothers in global psychiatric distress $(d=-0.39)$ although, by the end of the 3-month follow-up, mothers in both groups were approaching normative levels (i.e., $\mathrm{T}=$ 50) of psychiatric distress and low levels of depression.

Maternal substance use. After controlling for baseline scores, a marginally significant Group $\times$ Time interaction suggested that rate of heroin relapse differed for $\mathrm{MIO}$ and PE mothers (Table 6). MIO mothers showed a moderate decrease in proportion of heroin relapses $(d=-0.29)$ from month 2 to month 6 , whereas PE mothers showed a small increase $(d=0.21)$. Rates of relapse to other opioids remained low to nil in both groups across time, and there were no significant group differences over time. Rates of relapse to cocaine use remained similar for both groups across time.

Mother-child interactions. At the end of treatment, MIO children showed marginally higher levels of engagement with their mothers corresponding to a medium effect $(d=0.37$, see Table 5). There were no notable group differences in maternal sensitivity or dyadic reciprocity. At the end of the 3-month follow-up, although findings were not statistically significant, a small effect was found favoring MIO over PE mothers for sensitivity $(d=0.21)$ and dyadic reciprocity $(d$ $=0.21$ ). At the end of the 1-year follow-up, in comparison with PE mothers, MIO mothers showed significantly greater sensitivity $(d=0.46)$, MIO children showed marginally greater involvement with mothers $(d=0.28)$, and MIO dyads showed significantly greater reciprocity $(d=0.45)$.

Child attachment status. Table 5 shows that, at the end of treatment, there were no significant group differences in the percentage of children whose attachment status either remained secure or became more secure at posttreatment (Cramer $\phi=0.06)$.

\section{Moderation effects of addiction severity}

Maternal $R F$. Addiction severity did not moderate associations between treatment and mean RF at posttreatment $(t=$ $1.00, p=.32)$ or follow-up $(t=0.93, p=.11)$. The Addiction Severity $\times$ Treatment interaction was marginally significant for potential RF at posttreatment $\left(t=1.83, p=.07, f^{2}=\right.$ 0.04 ) but not significant at follow-up ( $t=1.70, p=.09$ ). The interaction pattern for potential RF at posttreatment (using actual change scores) is plotted in Figure 1. Further probing of individual slopes using simple regression analyses (Aiken \& West, 1991) revealed a significant slope for MIO mothers $\left(R^{2}=.16, \beta=0.16, p=.02\right)$ but not for PE mothers $\left(R^{2}=.01, \beta=-0.03, p=.69\right)$ indicating that, for MIO mothers only, as addiction severity increased, potential RF scores were higher, whereas for PE mothers, as addiction severity increased, potential RF scores did not vary. Simple $t$ tests were also conducted within condition to probe for differences in potential RF between low and high addiction severity levels (Figure 2). For PE mothers, the difference in potential RF between low $(M=4.61, S D=0.85)$ and high $(M=$ $4.42, S D=0.90)$ addiction severity groups was not statistically significant $(t=0.66, p=.51, d=0.15)$. For MIO mothers, however, the difference in potential RF scores between low $(M=4.53, S D=0.54)$ and high $(M=5.19$, $S D=0.54)$ addiction severity was significant with a large effect ( $t=2.98, p=.006, d=0.86)$. Together, these findings suggest that MIO served a protective function for mothers with more severe addiction in that they showed higher levels of potential RF at the end of treatment than their counterparts with low addiction severity. PE did not confer the same advantage.

Mother-child interactions. At posttreatment, addiction severity did not moderate associations between treatment and improvement in maternal sensitivity $(t=1.30, p=.20)$ or child involvement $(t=1.57, p=.12)$. However, the Addiction Severity $\times$ Treatment interaction was marginally significant for dyadic reciprocity $(t=1.77, p=.08)$. The interaction pattern is plotted in Figure 3 (using actual change scores). Further probing of individual slopes using simple regression analyses (Aiken \& West, 1991) revealed a significant slope for PE dyads $\left(R^{2}=.09, \beta=-0.14, p=.049\right)$ but not for MIO dyads 
Table 5. Results of primary treatment outcome analyses of covariance controlling for baseline scores

\begin{tabular}{|c|c|c|c|c|c|c|c|c|c|c|c|c|}
\hline & & & & & \multicolumn{8}{|c|}{ Follow-Up } \\
\hline & \multicolumn{4}{|c|}{ Post } & \multicolumn{4}{|c|}{3 Months } & \multirow{2}{*}{\multicolumn{4}{|c|}{12 Months }} \\
\hline & \multicolumn{2}{|c|}{ Estimated Mean $(S E)$} & \multirow[b]{2}{*}{$d^{a}$} & \multirow[b]{2}{*}{$F$} & \multicolumn{2}{|c|}{ Estimated Mean $(S E)$} & \multirow[b]{2}{*}{$d^{a}$} & \multirow[b]{2}{*}{$F$} & & & & \\
\hline & MIO & $\mathrm{PE}$ & & & MIO & PE & & & Estimated & Mean $(S E)$ & $d^{a}$ & $F$ \\
\hline \multicolumn{13}{|l|}{ Reflective functioning } \\
\hline Mean RF & $3.25(0.06)$ & $3.14(0.06)$ & 0.20 & 1.90 & $3.23(0.05)$ & $3.06(0.05)$ & 0.36 & $5.57 *$ & - & - & - & - \\
\hline Potential RF & $4.88(0.13)$ & $4.48(0.12)$ & 0.34 & $4.85^{*}$ & $4.82(0.06)$ & $4.36(0.06)$ & 0.82 & $32.91 * *$ & - & - & - & - \\
\hline \multicolumn{13}{|l|}{ Working model of the child } \\
\hline $\begin{array}{l}\text { Overall coherence } \\
\text { Curiosity box }\end{array}$ & $2.73(0.05)$ & $2.56(0.04)$ & 0.41 & $6.95^{*}$ & $2.78(0.04)$ & $2.58(0.04)$ & 0.54 & $12.07 * *$ & - & - & - & - \\
\hline Maternal sensitivity & $3.51(0.09)$ & $3.47(0.08)$ & 0.05 & 0.12 & $3.54(0.10)$ & $3.35(0.09)$ & 0.21 & 2.06 & $3.73(0.08)$ & $3.41(0.07)$ & 0.46 & $9.13 *$ \\
\hline Child involvement & $3.55(0.08)$ & $3.37(0.07)$ & 0.37 & $2.87 \dagger$ & $3.43(0.08)$ & $3.33(0.08)$ & 0.13 & 0.72 & $3.57(0.07)$ & $3.40(0.06)$ & 0.28 & $3.51 \dagger$ \\
\hline Dyadic reciprocity & $3.46(0.12)$ & $3.40(0.11)$ & 0.06 & 0.13 & $3.45(0.14)$ & $3.23(0.13)$ & 0.21 & 1.37 & $3.73(0.10)$ & $3.33(0.09)$ & 0.45 & $8.54 *$ \\
\hline Attachment status & & & $\phi^{c}$ & $\chi^{2}$ & & & & & & & & \\
\hline \multirow{2}{*}{$\begin{array}{l}\text { Remained or become more secure } \\
\text { Remained or become insecure or } \\
\text { disorganized }\end{array}$} & 64.3 & 58.1 & 0.06 & 0.24 & - & - & - & - & - & - & - & - \\
\hline & 35.7 & 41.9 & & & - & - & - & - & - & - & - & - \\
\hline
\end{tabular}

${ }^{a}$ Effect size $d: 0.20=$ small, $0.50=$ medium, $0.80=$ large $($ Cohen, 1988).

${ }^{b}$ Covariates included child age and sex.

${ }^{c}$ Cramer $\phi: 0.10=$ small, $0.30=$ medium, $0.50=$ large (source).

$* p<.05 . * * p<.01$. (two tailed)

$\dagger p<.10$. 


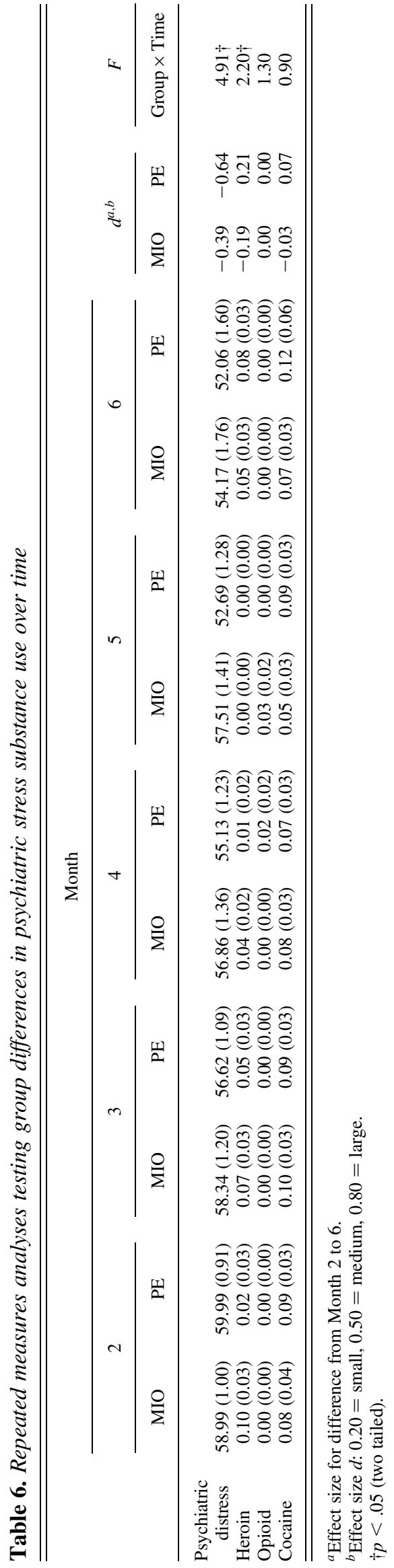

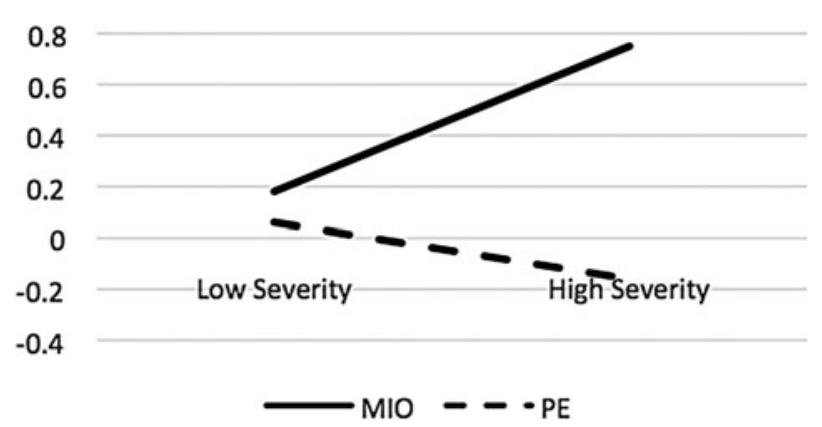

Figure 1. Addiction Severity $\times$ Treatment interaction for change in potential reflective functioning at posttreatment.

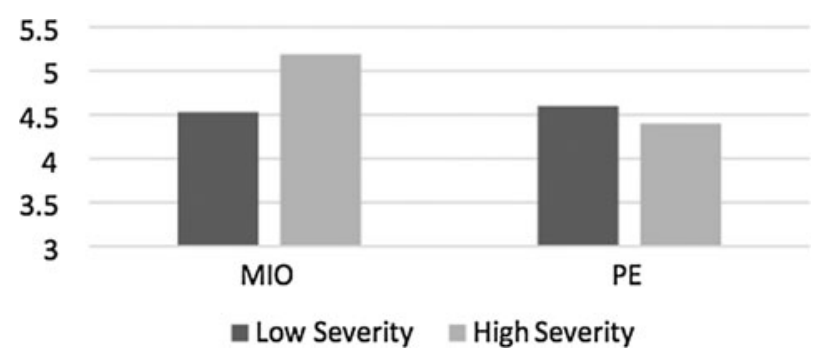

Figure 2. Potential reflective functioning scores at posttreatment for mothers with low and high addiction severity.

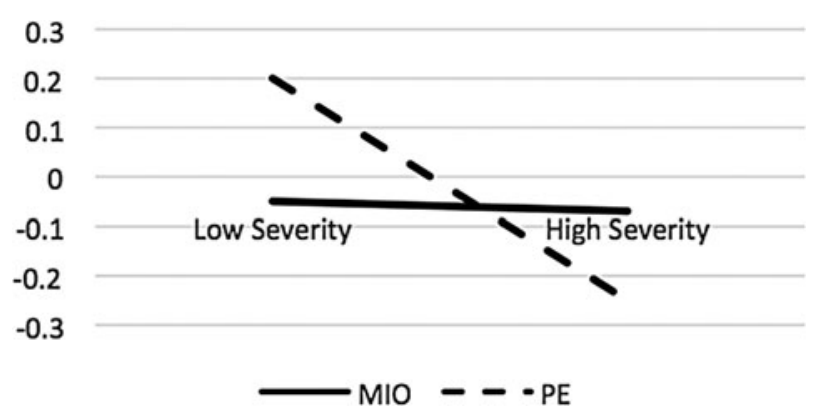

Figure 3. Dyadic reciprocity at posttreatment for mothers with low and high addiction severity.

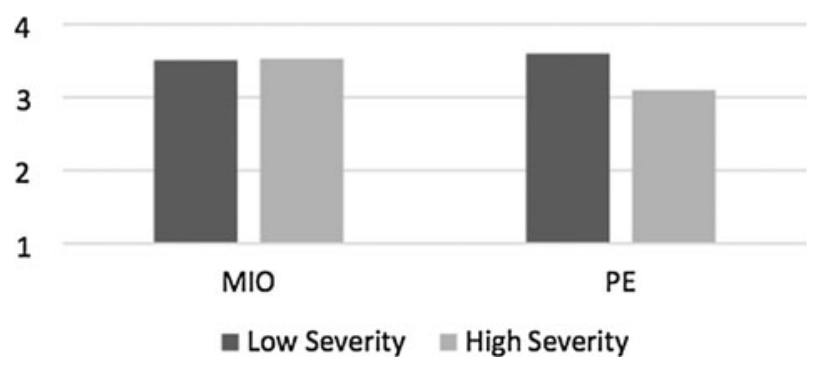

Figure 4. Dyadic reciprocity scores at posttreatment for mothers with low and high addiction severity. 
$\left(R^{2}=.01, \beta=0.04, p=.59\right)$ indicating that, for PE dyads only, as addiction severity increased, dyadic reciprocity became worse, whereas for MIO dyads, dyadic reciprocity did not vary with addiction severity. Simple $t$ tests were also conducted within condition to probe for differences in dyadic reciprocity scores between low and high addiction severity levels (Figure 4). For PE dyads, the difference in dyadic reciprocity between low $(M=3.60, S D=1.14)$ and high $(M=3.11, S D$ $=1.00)$ addiction severity groups was marginally significant and corresponded to a medium effect $(t=1.53, p=.13, d=$ 0.32 ). For MIO dyads, however, the difference in dyadic reciprocity between low $(M=3.51, S D=0.85)$ and high $(M=3.53, S D=0.80)$ addiction severity was nonsignificant $(t=-0.07, p=.94, d=0.02)$. Together, these findings suggest that MIO was protective for dyads where mothers reported more severe addiction in that their reciprocity was not compromised in the same way that it was for their PE counterparts at the end of treatment.

At the 3-month follow-up, addiction severity did not moderate associations between treatment and improvement in maternal sensitivity $(t=1.31, p=.20)$ or child involvement $(t=$ $1.48, p=.14)$. However, the Addiction Severity $\times$ Treatment interaction was marginally significant for dyadic reciprocity $\left(t=1.78, p=.08, f^{2}=0.04\right)$. The interaction pattern is plotted in Figure 5 (using actual change scores). Further probing of individual slopes using simple regression analyses (Aiken $\&$ West, 1991) revealed a significant slope for PE dyads $\left(R^{2}=.14, \beta=-0.16, p=.01\right)$ but not for MIO dyads $\left(R^{2}=.00, \beta=0.01, p=.88\right)$ indicating that, for PE dyads

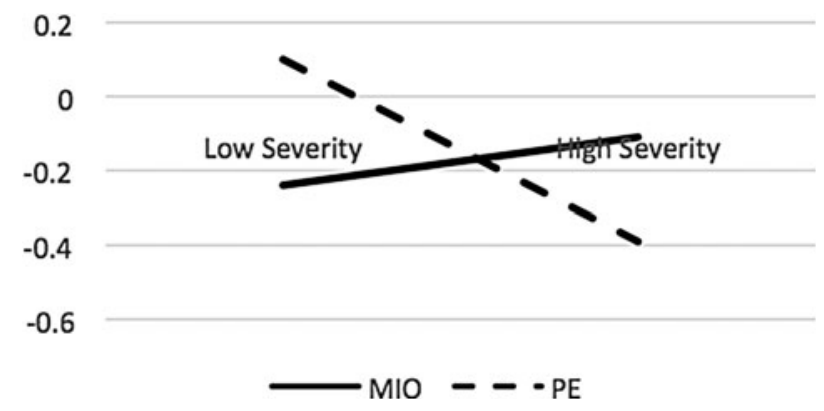

Figure 5. Dyadic reciprocity at 3-month follow-up for mothers with low and high addiction severity.

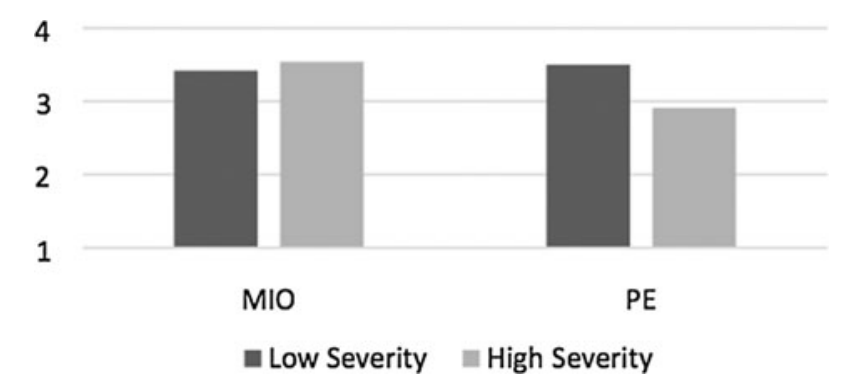

Figure 6. Dyadic reciprocity scores at 3-month follow-up for mothers with low and high addiction severity. only, as addiction severity increased, dyadic reciprocity became worse, whereas for MIO dyads, dyadic reciprocity did not vary with addiction severity. Simple $t$ tests were also conducted within condition to probe for differences in dyadic reciprocity scores between low and high addiction severity levels (Figure 6). For PE dyads, the difference in dyadic reciprocity between low $(M=3.50, S D=1.21)$ and high $(M=2.91, S D$ $=0.84$ ) addiction severity groups was marginally significant and corresponded to a medium effect $(t=1.90, p=.07, d=$ 0.40 ). For MIO dyads, however, the difference in dyadic reciprocity between low $(M=3.42, S D=0.79)$ and high $(M=$ $3.54, S D=0.84)$ addiction severity was nonsignificant $(t=$ $-0.45, p=.66, d=-0.10)$. Together, these findings suggest that MIO was protective for dyads where mothers reported more severe addiction in that their reciprocity was not compromised in the same way that it was for their PE counterparts at the 3-month follow-up.

12-month follow-up. At the 12-month follow-up, addiction severity significantly moderated associations between treatment and magnitude of change in maternal sensitivity $\left(t=2.12, p=.05, f^{2}=0.05\right)$, child involvement $(t=2.01$, $\left.p=.048, f^{2}=0.05\right)$, and dyadic reciprocity $(t=2.07, p=$ $\left..042, f^{2}=0.05\right)$. Because interaction patterns were similar across the three domains, only those for maternal sensitivity are plotted in Figure 7 (using actual change scores).

Maternal sensitivity. Further probing of individual slopes using simple regression analyses (Aiken \& West, 1991) re-

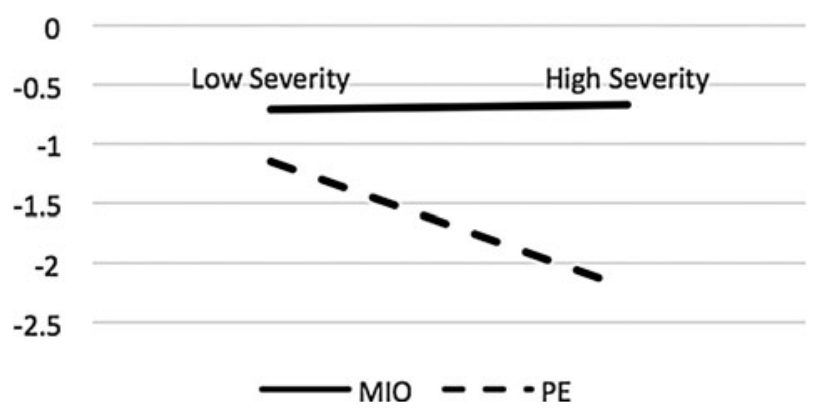

Figure 7. Addiction Severity $\times$ Treatment interaction for change in maternal sensitivity at 12 -month follow-up.

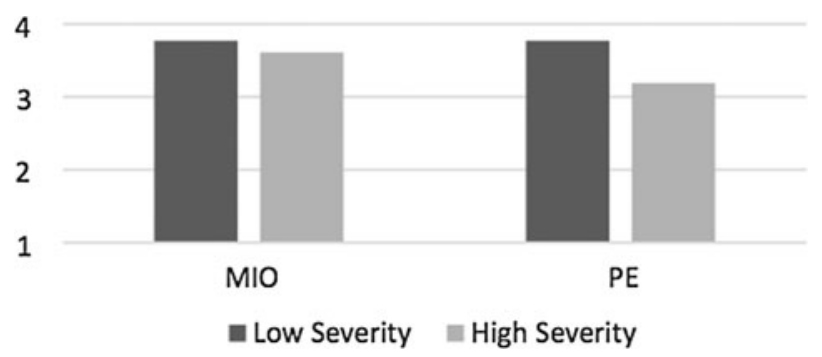

Figure 8. Maternal sensitivity scores at 12-week follow-up for mothers with low and high addiction severity. 
vealed a significant slope for PE mothers $\left(R^{2}=.15, \beta=-0.18\right.$, $p=.009)$ but not for MIO mothers $\left(R^{2}=.00, \beta=0.02, p=\right.$ .72) indicating that, for PE mothers only, as addiction severity increased, maternal sensitivity decreased, whereas for MIO mothers, as addiction severity increased, maternal sensitivity did not vary. Simple $t$ tests were also conducted within condition to probe for differences in maternal sensitivity scores between low and high addiction severity levels (Figure 8). For PE mothers, the difference in maternal sensitivity between low $(M=3.61, S D=0.71)$ and high $(M=3.19, S D=0.70)$ addiction severity was marginally significant and corresponded to a medium effect $(t=1.97, p=.055, d=0.42)$. For MIO mothers, the difference in maternal sensitivity scores between low $(M=3.77, S D=0.45)$ and high $(M=3.72, S D=0.36)$ addiction severity was not significant $(t=0.42, p=.68)$. These findings together suggest that addiction severity was (a) more likely to compromise rates of change in maternal sensitivity for PE mothers than for MIO mothers and (b) marginally more likely to compromise maternal sensitivity levels for $\mathrm{PE}$ mothers than for MIO mothers at the 12-month follow-up. Taken together, these findings suggest that MIO was protective for mothers who reported more severe addiction in that their sensitivity was not compromised in the same way that it was for their PE counterparts at the 12-month follow-up.

Child involvement. Further probing of individual slopes using simple regression analyses (Aiken \& West, 1991) revealed a significant slope for PE mothers $\left(R^{2}=.12, \beta=\right.$ $-0.15, p=.02)$ but not for MIO mothers $\left(R^{2}=.01, \beta=\right.$ $0.04, p=.56$ ) indicating that, for PE mothers only, as addiction severity increased, child involvement became lower, whereas for MIO mothers, as addiction severity increased, change in child involvement did not vary. Simple $t$ tests were also conducted within condition to probe for differences in child involvement scores between low and high addiction severity levels. For PE mothers, the difference in child involvement between low $(M=3.49, S D=0.63)$ and high $(M=3.32, S D=0.37)$ addiction severity was not significant and corresponded to a small effect $(t=1.15, p=.26, d=$ $0.23)$. For MIO mothers, the difference in child involvement between low $(M=3.53, S D=0.45)$ and high $(M=3.61$, $S D=0.34)$ addiction severity was also nonsignificant $(t=$ $-0.59, p=.56, d=-0.14)$. These combined findings suggest that MIO was protective for children of mothers who reported more severe addiction in that their involvement was not compromised in the same way that it was for their PE counterparts at the 12-month follow-up.

Dyadic reciprocity. Probing of individual slopes using simple regression analyses (Aiken \& West, 1991) revealed a significant slope for PE mothers $\left(R^{2}=.13, \beta=-0.16, p=.015\right)$ but not for MIO mothers $\left(R^{2}=.01, \beta=0.03, p>.10\right)$ indicating that, for PE dyads only, as addiction severity increased, dyadic reciprocity became worse, whereas for MIO dyads, as addiction severity increased, dyadic reciprocity did not vary. Simple $t$ tests were also conducted within condition to probe for differ- ences in child involvement scores between low and high addiction severity levels. For PE mothers, the difference in dyadic reciprocity between low $(M=3.51, S D=0.99)$ and high $(M$ $=3.09, S D=0.67$ ) addiction severity was marginally significant and corresponded to a medium effect $(t=1.69, p=.095, d$ $=0.35)$. For MIO mothers, the difference in dyadic reciprocity between low $(M=3.76, S D=0.59)$ and high $(M=3.77, S D=$ 0.53 ) addiction severity was nonsignificant $(t=-0.06, p=.95$, $d=-0.01)$. These findings suggest that MIO was protective for dyads where mothers reported more severe addiction in that their dyadic was not compromised in the same way that it was for their PE counterparts at the 12-month follow-up.

\section{Child attachment classification}

Compared to the base model, the full tested model (with main effects and interaction terms entered in a single block) significantly improved explanatory power $\left(\chi^{2}=4.37, p=.037\right)$, increasing prediction accuracy from $61 \%$ to $68 \%$. The Hosmer and Lemeshow test indicated good model fit $\left(\chi^{2}=\right.$ $6.06, p=.53$ ), and the Nagelkerke $R^{2}$ of .15 suggested that the full model explained $15 \%$ of the variance in attachment status change. As shown in Table 7, the odds ratio for the interaction effect indicated that, for each point of increase in MIO mothers' addiction severity, the odds of the target child's attachment status either remaining or becoming more secure doubled. Chi-square analyses were then conducted

Table 7. Results of binary logistic regression analysis testing moderator effects of addiction severity on change in attachment classification from pre- to posttreatment

\begin{tabular}{lrlc}
\hline \hline \multicolumn{1}{c}{ Variable } & \multicolumn{1}{c}{$B$} & Wald & $\operatorname{Exp}(\mathrm{B})$ \\
\hline Condition (MIO = 1, PE =0) & 0.24 & 0.17 & 1.27 \\
Addiction severity & -0.06 & 0.11 & 0.94 \\
Condition $\times$ Addiction Severity & 0.62 & $3.90^{*}$ & 1.87 \\
\hline \hline
\end{tabular}

$* p<.05$ (two tailed).

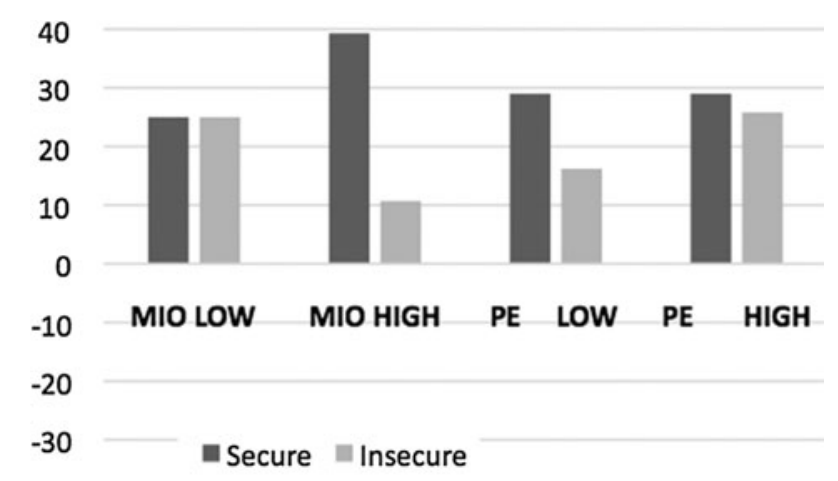

Figure 9. Percentage of children whose attachment status remained or approached secure versus insecure by condition and addiction severity (high vs. low). 
to probe for differences in percentage of children whose attachment status remained or became secure between low versus high addiction severity levels within condition (Figure 9). Within PE, $64.3 \%$ of children whose mothers' addiction severity was low and $52.9 \%$ of children whose mothers' addiction severity was high either remained secure or moved toward greater security at the end of treatment, and this difference was nonsignificant $\left(\chi^{2}=0.41\right.$, Fisher exact test $p=.72$, Cramer $\phi=0.11$ ) and corresponded to a small effect. Within MIO, $50 \%$ of children whose mothers' addiction severity was low and $78.6 \%$ of children whose mothers' addiction severity was high either remained secure or moved toward greater security at the end of treatment. Although this difference was nonsignificant $\left(\chi^{2}=2.49\right.$, Fisher exact test $p=.24$, Cramer $\phi=0.30$ ), the corresponding effect size is considered medium. Together, these findings suggest that MIO served a protective function for children of mothers with more severe addiction in that they showed more maintenance of or improvement toward a secure attachment classification. PE did not confer this same advantage for children of mothers with more severe addiction.

\section{Discussion}

In this paper, we reported results from the second randomized clinical trial testing the efficacy of MIO, a 12-session manualized individual mentalization-based therapy for mothers enrolled in substance abuse treatment. MIO was compared with $\mathrm{PE}$, a manualized individual psychoeducational intervention that was comparable to MIO in terms of treatment dose, individualized approach, and the chance to form a working alliance, but provided psychoeducation about developmental guidance and parenting strategies. The aims of the study were to (a) determine whether a mentalization-based approach would lead to better parental RF and representation coherence, reduction in psychiatric distress and relapse to substance use, better parent-child interactions, and more secure child attachment and (b) examine the potential protective role of MIO in relation to the impact of addiction severity on parenting and children's well-being. The implications of our findings for clinical work, and prevention and attachment science, are discussed in turn below.

\section{Implications for clinical interventions}

Maternal RF and representations. Mothers in MIO showed significantly greater potential for RF by the end of the 12-session treatment and significantly greater overall RF by the end of the 3-month follow-up. It is important that at posttreatment and 3-month follow-up, MIO mothers' potential RF capacity approached the benchmark score of 5, indicating an adequate capacity to recognize and make sense of mental (especially affective) experiences. These findings are consistent with those reported in the first randomized trial (Suchman et al., 2011). Likewise, at the end of treatment and at the 3-month follow-up, mental representations of the child and the care- giving relationship were significantly more coherent in mothers who received MIO. In other words, MIO mothers were more likely to express greater openness to their children's unique personalities and emotional needs and to have more realistic and balanced views of their children's capacities and personality characteristics. At both time points, MIO mothers' scores were approaching the benchmark of 3 , which is considered average. Together, these findings indicate the strong potential of relatively brief-but-intensive mentalization-based interventions for addressing the psychological deficits (e.g., impulsivity, emotional reactivity, and low distress tolerance) associated with chronic substance use and maladaptive parenting. The findings also underscore the importance of providing mentalization-based interventions for mothers concomitantly with their addiction treatment so that emotional regulation challenges inherent in the demands of being a parent in recovery can be targeted as they arise. Moreover, it seems reasonable to expect that, as mothers' mentalizing capacities improve, the transmission of this capacity to their young children is more likely.

MIO also proved to be protective for mothers with greater addiction severity at posttreatment. We were surprised that these mothers showed higher levels as well as greater improvement in potential RF than their counterparts with low addiction severity. We expected that mothers with greater addiction severity would have more to gain from an intervention focused on mentalizing but were puzzled as to why their potential RF scores were higher (i.e., RF score of 5.19 vs. 4.53). It may be that MIO is best suited to mothers with the most chronic and severe substance use disorders, whereas a combined focus on mentalization and developmental guidance might work better for mothers with less severe addiction problems.

Maternal psychiatric symptoms and substance use. Contrary to expectations, PE mothers showed a faster rate of decline in psychiatric distress than MIO mothers, although by the end of the 3-month follow-up, both groups had moved out of the clinically significant and into the normal range. In retrospect, it may be that asking mothers to pay closer attention to stressful situations and concomitant affective experiences results in slower alleviation of psychiatric distress. In the first trial (see Suchman et al., 2012), improvement in maternal depression was found to have a unique impact on caregiving behavior (even after improvement in maternal RF was taken into account). It will be important to examine further whether rate of decline in psychiatric distress might influence response to MIO.

A large majority of mothers in both conditions (at least 90\%) were not using any substances during the study, and this is likely due to their ongoing enrollment in outpatient addiction treatment. However, among the $10 \%$ of MIO mothers who acknowledged using heroin at the beginning of the study, there was a notable decline in relapse rate, whereas $\mathrm{PE}$ mothers' heroin relapse rate increased from $2 \%$ to $8 \%$ by the end of the 3-month follow-up. In light of recent increases 
in heroin use among adolescents and young adults in the United States, this finding has important implications, not only for parenting interventions but also for addiction treatment more broadly. Further examination of mechanisms of action are needed to determine whether improvement in mentalizing capacity leads to a reduction in relapse. If this turns out to be the case, introducing mentalization-based approaches to addiction treatment more broadly may help to improve relapse rates in the general treatment population. Current research (e.g., Rutherford, Potenza, \& Mayes, 2013) examining caregiving-related triggers, cravings, and relapse may also help identify the mechanisms by which mentalization-based therapy leads to better abstinence rates. Mechanisms notwithstanding, the benefits MIO mothers experienced in heroin abstinence further underscores the importance of providing mentalization-based parenting interventions and addiction treatment concomitantly. It is also critically important to recognize the potential risk in providing parenting programs that fail to address the unique emotional stressors associated with chronic addiction and parenting as evidenced by the small increase in heroin relapse rates despite PE's equivalence to MIO in all aspects (e.g., duration, intensity, supervision quality, and chance to form a secure alliance) except for the mentalizing focus.

Mother-child interactions. Small improvements were observed in MIO children's involvement at posttreatment and in MIO mothers' sensitivity and dyadic reciprocity at the 3month-follow-up. However, the greatest differences for MIO did not emerge until the 12-month follow-up when MIO mothers and children showed significantly better scores on all three relational indices (maternal sensitivity, child involvement, and dyadic reciprocity). It may take more time than we originally expected for changes in RF and maternal representations to reach the behavioral level for mothers and children. Future examination of the mediating effects of improved RF and representations in response to $\mathrm{MIO}$ will help clarify its mechanisms of change. It may also be important to consider mentalization-based therapy as the first in a series of steps that progress toward developmental and behavioral guidance as mothers become open and ready for constructive suggestions. Further research on alternative intervention progression may help clarify whether behavioral change can be accelerated. Likewise, engaging mothers in mentalization-based interventions earlier in their addiction recovery might also help prevent entrenchment in maladaptive parenting habits.

We also found strong evidence that MIO served a protective function for mothers with more severe addictions and their children against poorer interaction outcomes. For dyadic reciprocity at the 3-month follow-up and for all three motherchild interaction indices (maternal sensitivity, child involvement, and dyadic reciprocity) at the 12-month follow-up, we generally found a similar pattern showing that, when mothers with more severe addictions were assigned to PE, the chance for better quality relationships was diminished.
Again, we believe this finding indicates the potential risk, particularly for mothers with chronic substance abuse histories, in providing parenting programs that fail to address the unique emotional stressors associated with chronic addiction and parenting.

Child attachment status. At the end of treatment, $64.3 \%$ of MIO children and $58.1 \%$ of PE children either retained their secure status or moved toward a more secure status, and $35.7 \%$ of $\mathrm{MIO}$ children and $41.9 \%$ of PE children retained their insecure status or moved toward a less secure status. Although group differences were not significant, these data may signal that, with longer exposure to $\mathrm{MIO}$, group differences might be more pronounced. It is also possible that attachment status was measured prematurely. Like the results from the curiosity box, attachment status might not change until changes in maternal RF and representation have time to consolidate. In future work, we are planning to assess attachment status at follow-up visits as well.

Children of mothers with greater addiction severity were twice as likely to remain secure or become more secure in their attachment status if their mothers were assigned to MIO. Again, these findings suggest that children cared for by mothers with histories of severe addiction are more likely to benefit in terms of attachment security when their mothers receive a mentalization-based parenting intervention.

\section{Implications for intervention and attachment research}

Findings from this and our previous randomized trial (Suchman et al., 2010, 2011, 2012) highlight the importance of carefully considering mechanisms of change when developing and evaluating interventions for parent populations at the far end of the psychosocial risk spectrum (e.g., parents with chronic addiction and psychiatric disorders). Interventions that directly target change in parenting behavior through psychoeducational instruction and hands-on parent coaching have generally worked well to prevent escalation of conduct problems in children whose parents are experiencing moderate environmental stressors and psychosocial challenges (Sanders, Markie-Dadds, Tully, \& Bor, 2000; Webster-Stratton \& Reid, 2010). However, to date, none of the evidencebased behavior-targeting interventions have demonstrated promise for parents at the extreme end of the risk spectrum who often leave these interventions prematurely and accumulate experiences of frustration and failure as they continue to draw the attention of child welfare agencies. Our research suggests that, for parents who have more profound difficulties tolerating stress, regulating emotions, and managing impulses, targeting these psychological deficits in a brief yet intensive individualized therapy that focuses on ameliorating these issues (i.e., strengthening the mentalizing capacity) may be a more effective route to improving parent-child relationships and preventing the intergenerational transmission of addiction psychopathology. Although it is too early to draw conclusions (e.g., tests of mechanisms need to be replicated), 
our evidence suggests that targeting a parent's psychosocial deficits within the context of parenting may help free the parent psychologically and emotionally to engage with her child in a more mutually rewarding and enjoyable manner that ultimately benefits the child's experience of emotional security and self-agency.

The concept of mentalization and its observable manifestation in RF appears to be a critical mechanism in the transmission of secure attachment across generations that requires further study in high-risk populations. Unlike other complex psychodynamically grounded constructs (e.g., ego development), mentalization has been operationalized for empirical research (Luyten et al., 2009; Slade et al., 2003) and has strong theoretical grounding in and empirical association with adult and child attachment (see Katznelson, 2014, for a review). As a measurable human psychological capacity that is increasingly considered critical to interpersonal functioning (see, e.g., Gabbard, 2010), and central in many forms of psychopathology, its relevance to intervention and attachment research will likely continue to grow. Ironically, it is a capacity that has been studied more extensively in children (i.e., theory of mind research) than in adults or parents. Our findings along with the others' (e.g., Pajulo et al., 2012; Sadler et al., 2013) point to the critical role of parental mentalizing and RF starting during (and even before) pregnancy, as parents' representations of their unborn infants become activated and begin to inform many aspects of their decisions and behaviors.

\section{Conclusions and future directions}

Substance addiction has a profound impact on adults who find themselves in the parenting role caring for young children and even more profoundly on the young children themselves. Intervention with this vulnerable and often disenfran-

\section{References}

Aiken, L. S., \& West, S. G. (1991). Multiple regression: Testing and interpreting interactions. Thousand Oaks, CA: Sage.

Ainsworth, M. D. S., Blehar, M. C., Waters, E., \& Wall, S. (1978). Patterns of attachment: A psychological study of the strange situation. Hillsdale, NJ: Erlbaum.

Allen, J. G., Fonagy, P., \& Bateman, A. (2008). Mentalizing in clinical practice (1st ed.). Arlington, VA: American Psychiatric Publishing.

Bateman, A. W., \& Fonagy, P. (2012). Handbook of mentalizing in mental health practice (1st ed.). Arlington, VA: American Psychiatric Publishing.

Bayley, N. (2006). Bayley Scales of Infant and Toddler Development Screening Test (3rd ed.). San Antonio, TX: Harcourt Assessment.

Buckley, C. (2009, September 25). In the city, highly productive drug mills. New York Times. Retrieved from http://www.nytimes.com.

Burns, K. A., Chethik, L., Burns, W. J., \& Clark, R. (1997). The early relationship of drug abusing mothers and their infants: An assessment at eight to twelve months of age. Journal of Clinical Psychology, 53, 279-287.

Cassidy, J., \& Marvin, R. S., with the MacArthur Attachment Working Group. (1992). Attachment organization in preschool children: Coding guidelines (4th ed.). Unpublished manuscript, University of Virginia.

Choi, S., \& Ryan, J. (2007). Co-occurring problems for substance abusing mothers in child welfare: Matching services to improve family reunification. Children and Youth Services Review, 29, 1395-1410. chised and stigmatized population is very challenging and requires theoretically grounded approaches that take the unique psychosocial consequences of addiction into account. Here we reported on one such approach that is grounded in attachment and mentalization theory and builds on neuroscientific evidence showing that chronic substance use coopts the same neural circuitry recruited during parenting activities and likely makes the parenting experience more stressful and less enjoyable. The findings reported here support the importance of addressing a mother's subjective experience of distress in the parenting role while she is in recovery and before addressing her parenting behaviors with her young children. The results bring to mind the oxygen mask metaphor where airline staff instruct parents to place their oxygen mask first on themselves before fixing their child's mask. Children's unmet needs cannot be addressed without first meeting the needs of their primary caregivers, who are, most typically, their mothers.

Mentalization-based therapy for mothers has now shown promise for improving maternal RF and caregiving quality in two randomized clinical trials. The next step in our research program (currently under way) involves training addiction counselors to deliver MIO and PE with fidelity and testing whether MIO's efficacy holds when delivered by addiction counselors in a community-based setting. We are hopeful that training addiction counselors in mentalization-based therapy, child development, and effective parenting strategies will help stem the gap between child guidance and addiction services that parents in recover often fall through. So far, our experience shows us that addiction counselors welcome the additional training and are very well poised to provide this additional support to parents in recovery. Forthcoming results of our Stage III community-based randomized clinical trial will provide the needed empirical evidence that will either support or refute this hypothesis.

Cohen, J. (1988). Statistical power analysis for the behavioral sciences (2nd ed.). Hillsdale, NJ: Erlbaum.

Cohen, J., \& Cohen, P. (1983). Applied multiple regression/correlation analysis for the behavioral sciences. Hillsdale, NJ: Erlbaum.

DeCoste, C., Dalton, R., de las Heras Kuhn, L., \& Dennehy, L. (2010). Parent Education: An individualized psycho-educational program for mothers of children under 5 years of age. New Haven, CT: Yale University School of Medicine.

Department of Health and Human Services. (1999). Blending perspectives and building common ground: A report to congress on substance abuse and child protection. Washington, DC: Author.

Derogatis, L. R. (1993). Brief Symptom Inventory: Administration, scoring, and procedures manual (3rd ed.). Minneapolis, MN: National Computer Systems.

Fals-Stewart, W., O-Farrell, T. J., Freitas, T. T., McFarlin, S. K., \& Rutigliano, P. (2000). The Timeline Followback reports of psychoactive substance use by drug-abusing patients: Psychometric properties. Journal of Consulting and Clinical Psychology, 68, 134-144.

Feldman, R. (1998). Coding interactive behavior manual. Tel Aviv: Bar-Ilan University.

Fonagy, P., Steele, M., Steele, H., Leigh, T., Kennedy, R., Mattoon, G., \& Target, M. (1995). Attachment, the reflective self, and borderline states: The predictive specificity of the Adult Attachment Interview and pathological emotional development. In S. Goldberg, R. Muir, \& J. Kerr 
(Eds.), Attachment theory: Social, developmental, and clinical perspectives (pp. 233-278). Hillsdale, NJ: Analytic Press.

Gabbard, G. O. (2010). Long-term psychodynamic psychotherapy: A basic text (2nd ed.). Arlington, VA: American Psychiatric Publishing.

Gottman, J. M., \& Rushe, R. H. (1993). The analysis of change: Issues, fallacies, and new ideas. Journal of Consulting and Clinical Psychology, 61, 907-910.

Grant, T., Huggins, J., Graham, J. C., Ernst, C., Whitney, N., \& Wilson, D. (2011). Maternal substance abuse and disrupted parenting: Distinguishing mothers who keep their children from those who do not. Children and Youth Services Review, 33, 2176-2185.

Greenfield, S. F., Back, S. E., Lawson, K., \& Brady, K. T. (2010). Substance abuse in women. Psychiatric clinics of North America, 33, 339-355.

Hans, L. L., Bernstein, V. J., \& Henson, L. G. (1999). The role of psychopathology in the parenting of drug-dependent women. Development and Psychopathology, 11, 957-977.

Harrison, P. L., Kaufman, A. S., Kaufman, N. L., Bruininks, R. H., Rynders, J., Ilmer, S., . . Cicchetti, D. V. (1990). AGS Early Screening Profiles. Journal of Psychoeducational Assessment, 13, 101-104.

Hoffman, K. T., Marvin, R. S., Cooper, G., \& Powell, B. (2006). Changing toddlers' and preschoolers' attachment classifications: The Circle of Security Intervention. Journal of Consulting and Clinical Psychology, 74, 1017-1026.

Jones, R. G. (2008, January 13). Heroin's hold on the young. New York Times. Retrieved from http://www.nytimes.com

Katznelson, H. (2014). Reflective functioning: A review. Clinical Psychology Review, 34, 107-117.

Kaufman, A. S., \& Kaufman, N. L. (1990). Kaufman Grief Intelligence Test. Circle Pines, MN: American Guidance Service.

Kerwin, M. E. (2005). Collaboration between child welfare and substanceabuse fields: Combined treatment programs for mothers. Journal of Pediatric Psychology, 30, 581-597.

Luyten, P., Mayes, L. C., Sadler, L., Fonagy, P., Nicholls, S., Crowley, M., .. Slade, A. (2009). The Parental Reflective Functioning Questionnaire-1 (PRFQ-1). Unpublished manuscript, University of Leuven.

Mayes, L., Carter, A., \& Stubbe, D. (1993). Infant differences in exploratory behavior in the second year of life. Infant Behavior and Development, 16, 269-284.

Miller, M. D. (1995). Kaufman Brief Intelligence Test. In J. C. Conoley \& J. C. Impara (Eds.), The twelfth mental measurements yearbook (pp. 533534). Lincoln, NE: Buros Institute of Mental Measurements.

Pajulo, M., Pyykkönen, N., Kalland, M., Sinkkonen, J., Helenius, H., Punamäki, R., \& Suchman, N. (2012). Substance abusing mothers in residential treatment with their babies: Importance of focusing in pre- and postnatal maternal reflective functioning. Infant Mental Health Journal, 33, 70-81.

Rogosa, D. R. (1995). Myths and methods: "Myths about longitudinal research," plus supplemental questions. In J. M. Gottman (Ed.), The analysis of change (pp. 3-65). Hillsdale, NJ: Erlbaum.

Rutherford, H. J. V., Potenza, M. N., \& Mayes, L. C. (2013). The neurobiology of addiction and attachment. In N. E. Suchman, M. Pajulo, \& L. Mayes (Eds.), Parenting and substance abuse: Developmental approaches to intervention (pp. 3-23). New York: Oxford University Press.

Rutherford, H. J. V., Williams, S. K., Moy, S., Mayes, L. C., \& Johns, J. M. (2011). Disruption of maternal parenting circuitry by addictive process: Rewiring of reward and stress symptoms. Frontiers in Psychiatry, 2, 37.

Sadler, L. S., Slade, A., Close, N., Webb, D. L., Simpson, T., Fennie, K., \& Mayes, L. C. (2013). Minding the Baby: Enhancing reflectiveness to improve early health and relationship outcomes in an interdisciplinary home visiting program. Infant Mental Health Journal, 34, 391-405.

Sanders, M. R. (1999). Triple P-Positive Parenting Program: Towards an empirically validated multilevel parenting and family support strategy for the prevention of behavior and emotional problems in children. Clinical Child and Family Psychology Review, 13, 71-90.

Sanders, M. R., Markie-Dadds, C., Tully, L. A., \& Bor, W. (2000). The Triple P-Positive Parenting Program: A comparison of enhanced, standard, and self-directed behavioral family intervention for parents of children with early onset conduct problems. Journal of Consulting and Clinical Psychology, 68, 624-640.

Schafer, J. L., \& Graham, J. W. (2002). Missing data: Our view of the state of the art. Psychological Methods, 7, 147-177.

Seelye, K. Q. (2015, July 11). Obituaries shed euphemisms to chronicle toll of heroin. New York Times. Retrieved from http://www.nytimes.com.

Slade, A. (2005). Parental reflective functioning: An introduction. Attachment and Human Development, 7, 269-281.

Slade, A., Aber, J. L., Berger, B., Bresgi, I., \& Kaplan, M. (2003). The Parent Development Interview - Revised. Unpublished manuscript, City University of New York.

Sobell, L. C., \& Sobell, M. B. (1992). Timeline Follow-Back: A technique for assessing self-reported alcohol consumption. In R. Z. Litten \& J. Allen (Eds.), Measuring alcohol consumption: Psychosocial and biological methods (pp. 4-72). Totowa, NJ: Humana Press.

Sobell, L. C., \& Sobell, M. B. (1996). Timeline Followback user's guide: A calendar method for assessing alcohol and drug use. Toronto: Addiction Research Foundation.

Solomon, J., \& George, C. (2008). The measurement of attachment security in infancy and childhood. In J. Cassidy \& P. R. Shaver (Eds.), Handbook of attachment: Theory, research, and clinical applications (2nd ed., pp. 383-416). New York: Guilford Press.

Strathearn, L. (2011). Maternal neglect: Oxytocin, dopamine and the neurobiology of attachment. Journal of Neuroendocrinology, 23, 1054-1065.

Strathearn, L., Fonagy, P., Amico, J., \& Montague, P. R. (2009). Neuropsycopharmacology, 34, 2655-2666.

Suchman, N. E., \& Bers, S. A. (2015). Mothering from the Inside Out: A mentalization-based intervention for mothers in substance use treatment. New Haven, CT: Yale University School of Medicine.

Suchman, N. E., DeCoste, C., McMahon, T., Rounsaville, B., \& Mayes, L. (2011). The Mothers and Toddlers Program, an attachment-based parenting intervention for substance-using women: Results at 6-week follow-up in a randomized clinical pilot. Infant Mental Health Journal, 32, 427-449.

Suchman, N. E., DeCoste, C., Ordway, M., \& Bers, S. (2013). Mothering from the Inside Out: A mentalization-based individual therapy for mothers with substance use disorders. In N. Suchman, M. Pajulo, \& L. Mayes (Eds.), Parenting and substance addiction: Developmental approaches to intervention (pp. 407-433). New York: Oxford University Press.

Suchman, N. E., DeCoste, C., Rosenberger, P., \& McMahon, T. J. (2012) Attachment-based intervention for substance using mothers: A preliminary test of the proposed mechanisms of change. Infant Mental Health Journal, 33, 360-371.

Suchman, N. E., Pajulo, M., DeCoste, C., \& Mayes, L. C. (2006). Parenting interventions for drug dependent mothers and their young children: The case for an attachment-based approach. Family Relations, 55, 211-226.

Suchman, N. E., Rosenberger, P., \& DeCoste, C. (2006). The Mothers and Toddlers Program: Therapist adherence and coding procedures manual. New Haven, CT: Yale University School of Medicine.

Suchman, N. E., Rosenberger, P., \& DeCoste, C. (2010). The Revised MIO/ PE Therapist Adherence Rating Scale and Coding Procedures manual. New Haven, CT: Yale University School of Medicine.

Toth, S. L., Rogosch, F. A., Manly, J. T., \& Cicchetti, D. (2006). The efficacy of toddler-parent psychotherapy to reorganize attachment in the young offspring of mothers with major depressive disorder: A randomized preventive trial. Journal of Consulting and Clinical Psychology, 74, 10061016

Webster-Stratton, C., \& Reid, M. J. (2010). The Incredible Years parents, teachers and children training series: A multifaceted treatment approach for young children with conduct problems. In J. Weisz \& A. Kazdin (Eds.), Evidence-based psychotherapies for children and adolescents (2nd ed., pp. 194-210). New York: Guilford Press.

Young, J. W. (1995). Kaufman Brief Intelligence Test. In J. C. Conoley \& J. C. Impara (Eds.), The twelfth mental measurement yearbook (pp. 534-535). Lincoln, NE: Buros Institute of Mental Measurements.

Zeanah, C. H., \& Benoit, D. (1993). Clinical applications of a parent perception interview in infant mental health. Infant Psychiatry, 3, 539-554. 